\title{
Molecular scale description of interfacial mass transfer in phase separated aqueous secondary organic aerosol
}

\author{
Mária Lbadaoui-Darvas ${ }^{1}$, Satoshi Takahama ${ }^{1}$, and Athanasios Nenes ${ }^{1,2}$ \\ ${ }^{1}$ School of Architecture, Civil and Environmetal Engineering, Swiss Federal Institute of Technology, Lausanne, 1015, \\ Switzerland \\ ${ }^{2}$ Institute of Chemical Engineering Sciences, Foundation for Research and Technology Hellas, Patras, Greece GR-26504
}

Correspondence: Athanasios Nenes (athanasios.nenes@epfl.ch), Satoshi Takahama (satoshi.takahama@epfl.ch)

\begin{abstract}
Liquid-liquid phase separated (LLPS) aerosol particles are known to exhibit increased CCN activity compared to well mixed ones due to a complex effect of low surface tension and non-ideal mixing. The relation between the two contributions as well as the molecular scale mechanism of water uptake in the presence of an internal interface within the particle is to date not fully understood. Here we present steered molecular dynamics simulation studies of water uptake by a vapor/hydroxicis-pinonic acid/water double interfacial system at $200 \mathrm{~K}$ and $300 \mathrm{~K}$. Simulated free energy profiles are used to map the water uptake mechanism and are decomposed into energetic and entropic contributions to highlight its main thermodynamic driving forces. Atmospheric implications are discussed in terms of gas/particle partitioning, intraparticle water redistribution timescales, and equilibrium saturation ratios of water vapor. Our simulations reveal a strongly temperature-dependent water uptake mechanism, whose most prominent features are determined by local extrema in conformational and orientational entropies near the organic/water interface which result in a reduced core uptake coefficient $\left(k_{o / w}=0.05\right)$ and a concentration gradient of water in the organic shell at the higher temperature, while their effect is negligible at $200 \mathrm{~K}$ due to the explicit temperature dependence of entropic terms in the free energy profiles. The concentration gradient, which is a molecular level manifestation of non-ideal mixing - suspected to be a major factor to increase LLPS CCN activity - is responsible for maintaining LLPS and low surface tension even at very high relative humidities, thus reducing critical supersaturations. Thermodynamic driving forces are rationalized to be generalizable across different compositions. The conditions under which single uptake coefficients can be used to to describe growth kinetics as a function of temperature in LLPS particles are described.
\end{abstract}

\section{Introduction}

Aerosol-cloud interactions constitute one of the most important sources of uncertainty in assessments of anthropogenic climate change (IPCC). The number, size and composition characteristics of aerosol influence the number of droplets that can form in cloudy updrafts, which in turn affect the microphysical evolution and radiative properties in a complex and intricate way up to the climate scale. Cloud droplets form upon a subset of aerosol, called cloud condensation nuclei (CCN), that become unstable and experience unconstrained growth in the supersaturated water vapor that develops in a cloudy updraft. The dynamics of water uptake on $\mathrm{CCN}$ is a critical process that influences the level of supersaturation that can develop in clouds (Raatikainen 
et al., 2013) and is affected by the interplay of gas-to-particle transport, interfacial mass transfer and diffusion in the particle phase. Water uptake by particles is controlled by the gas-to-particle transport timescale when the mean free path of gas phase molecules is smaller than the particle size. Particle phase diffusion dominates in glassy and semisolid aerosol, while interfacial mass transfer can be important when the particle size is comparable to or smaller than the mean free path of in the gas phase.

Limitations on particle growth by interfacial mass transfer can be expressed in terms of the mass accommodation coefficient $(\alpha)$, defined as the ratio of molecules absorbed by the particle over the total number of molecules colliding with the surface. $\alpha \sim 1$ for particles whose surface is composed of dominantly water (Clement et al., 1996; Morita et al., 2004; Voigtländer et al., 2007), while $\alpha$ can be very reduced for hydrophobic surfaces. For hydrophobic organic films (e.g.: long chained alcohol) model films $\alpha$ was found to be as low as $10^{-3}-10^{-5}$ from molecular dynamics (Takahama and Russell, 2011; Ergin and Takahama, 2016; Johansson et al., 2020) and experimental studies (Diveky et al., 2019; Johansson et al., 2020). Gas-to-particle partitioning on secondary organic aerosol (SOA), which constitutes a significant fraction of the total particulate matter on a global scale (Fuzzi et al., 2006), is usually characterized by $\alpha>0.1$ (Raatikainen et al., 2013; Julin et al., 2014; Krechmer et al., 2017). Modeling studies have long shown that reduced mass accommodation coefficients result in increased droplet number concentration in clouds, owing to the slow condensation of water vapor in the initial stages of cloud formation, which in turn elevates supersaturation and allows for more $\mathrm{CCN}$ to activate. For these kinetic delays to be important for climate simulations, the uptake coefficient needs to be less than 0.1 (Raatikainen et al., 2013). To date, although low values of $\alpha$ have been reported for select systems, analysis of droplet formation upon aerosol from a broad range of environments - even aerosol with large amounts of hydrophobic material (Moore et al., 2012; Raatikainen et al., 2013) has not indicated uptake coefficients below 0.1. $\alpha$ also depends on temperature in a system specific way (Li et al., 2001; Davidovits et al., 2004; Zientara et al., 2008; Davies et al., 2013; Roy et al., 2020). This is typically not accounted for by parcel models of cloudy updrafts, which tend use a single $\alpha$ value to simulate the entire range on which the temperature of a rising air parcel varies (Morales Betancourt and Nenes, 2014), which can lead to systematic errors, whose magnitude may depend on elevation.

While the single parameter description, which uses only the mass accommodation coefficient to describe gas-to-particle partitioning in atmospheric systems, is extremely widespread, in reality $\alpha$ is not unambiguously defined. A clear definition of the reference state - i.e., the state beyond which a water molecule is considered to have partitioned to the particle phase 50 - is unclear. The most commonly accepted definition of $\alpha$ uses adsorption at the particle surface or absorption by the first few molecular layers of the particle as a reference state, while a penetration depth-dependent definition (Shiraiwa and Pöschl, 2020) has also been proposed. The latter accounts for particle phase diffusion using an effective accommodation coefficient. This approach turns out to be sufficiently complex to describe water uptake by well mixed particles.

Typical values of $\alpha>0.1$ observed for SOA CCN suggest that they do not substantially exhibit kinetic delays in gas-toparticle partitioning. Nevertheless, increased CCN activity compared to the one predicted by the $\kappa$-Köhler theory has been reported for SOA in particular for liquid-liquid phase separated (LLPS) particles (Prenni et al., 2007; Pajunoja et al., 2015; Liu et al., 2018). LLPS occurs in deliquesced aerosol rich in SOA if the O:C ratio of the organics is lower than 0.8 (Song et al., 2012, 2017; Renbaum-Wolff et al., 2016). Particles formed by LLPS have either core-shell or partially engulfed morphopolies (Song et al., 2012; You et al., 2014; Gorkowski et al., 2020). LLPS organic shells are non-ideal mixed multilayers, potentially 

accommodation coefficient (Krieger et al., 2012; Davies et al., 2013). Increased CCN activity of LLPS particles may be due to i) reduced surface tension due to the high concentrations organic molecules at the surface of LLPS particles ; ii) surface adsorption (Pajunoja et al., 2015; Sareen et al., 2013) or iii) non-ideal mixing. Surface tension reduction is proven in laboratory (Song et al., 2012, 2017; Renbaum-Wolff et al., 2016) and field experiments (Facchini et al., 1999), and its role in enhancing CCN activity has been extensively discussed (Ruehl et al., 2012, 2016; Noziere, 2016; Ovadnevaite et al., 2017). The existence of a long-lived surface adsorbed states is unlikely when the outer phase has randomly oriented hydrophobic groups, due to the rapid formation of $\mathrm{H}$-bonds seen in MD simulations (Johansson et al., 2020), and was recently proved to be insignificant also by experiments (Liu et al., 2018). Recent studies point out the importance of non-ideal mixing in reduced CCN activity by introducing a concentration Flory-Huggins type of representation of the non-ideal Gibbs free energy of mixing in a $\kappa$-Köhler model (Petters et al., 2006; Liu et al., 2018). However, at the level of complexity of the Flory-Huggins theory it is not possible to explain how non-idealities appearing on a molecular scale can enhance CCN activity. The connection between the reduced surface tensions and non-ideal mixing effects has yet to be investigated. Molecular scale understanding of the manifestation of non-idealities in the particle structure can help establish this link and can be obtained almost uniquely from molecular simulations.

Both the ambiguities surrounding the definition of gas-to-particle partitioning in particles that are not well mixed, and the lack of detailed understanding of the effect of LLPS on cloud droplet growth and activation are closely tied to our limited knowledge about the molecular scale mechanism of the partitioning process, which has been mapped for only a handful of systems (Sakaguchi and Morita, 2012; Ergin and Takahama, 2016). Nanosecond timescales associated with interfacial mass transfer of a single water molecule to an aerosol particle (Bzdek and Reid, 2017) are conveniently studied by molecular dynamics (MD) simulations, using equilibrium (Bahadur and Russell, 2008), direct impinging (Vieceli et al., 2005; Takahama and Russell, 2011; Johansson et al., 2020) or umbrella sampling techniques (Sakaguchi and Morita, 2012; Ergin and Takahama, 2016). They have estimated near unity surface accommodation coefficients on pure water surfaces (Morita et al., 2004; Vieceli et al., 2005; Takahama and Russell, 2011) and reproduced reduced coefficients characteristic of hydrophobic surfaces (Sakaguchi and Morita, 2012; Ergin and Takahama, 2016; Miles et al., 2016; Johansson et al., 2020) as well as size dependence of uptake coefficient in salt nanoparticles (Bahadur and Russell, 2008). In direct impinging simulations, gas-phase molecules are launched with an initial velocity towards the bulk phase in several trials and the mass accommodation coefficient is simply estimated as the ratio of successful trials. This method requires additional techniques to identify the eventual fate of molecules that are adsorbed at the surface but desorbed later without entering the bulk liquid phase. Umbrella sampling (Torrie and Valleau, 1977) simulations are used to reconstruct the free energy profile of water uptake from a series equilibrium simulations with 90 the position of the gas phase molecule at different distances from the bulk, which is then converted to mass accommodation coefficient using the transition state theory (Sakaguchi and Morita, 2012). In its traditional formulation umbrella sampling used to study transfer through liquid surfaces artificially smoothes the effect thermal surface fluctuations due to the way in which averaging is performed (Darvas et al., 2013). A very recent study used well-tempered metadynamics to estimate equilibrium partitioning coefficients of volatile organics (von Domaros et al., 2020). In this work, an alternative approach based on steered 
molecular dynamics (Steered MD) (Park and Schulten, 2004) is proposed. It has been successful at exploring free energy profiles using out of equilibrium simulations along distance-related reaction coordinates in biophysical contexts (Allen et al., 2014, e.g.).

This paper presents a molecular simulation study aimed at revealing the mechanism of water uptake in a vapor/hydroxy-cispinonic acid/water double interfacial system at two temperatures, characteristic of the planetary boundary layer and the upper troposphere. Free energy profiles of water uptake are generated using steered MD simulations, and are used to describe the temperature dependence of the water uptake mechanism. Thermodynamic driving forces are identified by decomposing the free energy profiles into entropic and energetic contributions, and the causality between exact molecular scale representation of non-ideal mixing thermodynamics and observed increased CCN activities is inferred from the thermodynamic description. Knowing the molecular-scale mechanism of water uptake, the usability of a single mass accommodation coefficient to describe gas to particle partitioning is assessed by modeling characteristic timescales and concentration distributions in the organic shell. Finally, different scenarios through which LLPS affects cloud droplet activation and growth are identified and tested in the framework of the Köhler-theory.

\section{Methods}

\subsection{Technical background}

The molecular scale mechanism of water uptake is studied through the analysis of free energy profiles. This approach provides a comprehensive overview of the gas-to-particle partitioning process. Molecular simulation methods to calculate free energy profiles, among them umbrella sampling, which is the traditional approach to obtain transfer coefficients (Sakaguchi and Morita, 2012) and steered molecular dynamics, which is used in this study, rely on forcing the system to follow a pathway along an aptly chosen set of reaction coordinates that provide a low-dimensional representation of the physical process. In umbrella sampling (Torrie and Valleau, 1977), the reaction coordinate space is mapped in a set of consecutive quasi-equilibrium simulations, with the reaction coordinates restrained at a different value in each simulation. Quasi-equilibrium methods (Sakaguchi and Morita, 2012) provide a free energy estimate that artificially averages out effects of surface fluctuations (as a result of the method used to average the force as a function of the reaction coordinate) (Darvas et al., 2013; Braga et al., 2016; Klug et al., 2018) whose spatial and temporal scales are similar to those of a typical atmospheric water uptake event.

In steered MD (Park and Schulten, 2004), the system is pulled along the reaction coordinate space with the help of an external harmonic bias at a constant finite velocity or with a constant finite force in several parallel realisations. Jarzynski's equality (Jarzynski, 1997) is used to estimate the free energy from the work profiles collected in each non-equilibrium simulation:

$e^{-\beta \Delta G}=\left\langle e^{-\beta W}\right\rangle$,

where $\beta=1 / k_{B} T$ and $k_{B}$ is the Boltzmann constant and $T$ is the temperature. A formal proof of Jarzinsky's equality for finite size systems, coupled to an external heat reservoir (a typical model of a canonical MD simulation) can be found elsewhere (Cuendet, 2006; Schöll-Paschinger and Dellago, 2006). Besides free energy differences, steered MD can also be used to re- 
construct free energy profiles using different reweighting schemes (Gore et al., 2003). The reweighting is necessary as every "pulling simulation" relies on attaching a harmonic spring to the molecular system in the direction of the reaction coordinate $(s(\mathbf{x}, t)))$. This signifies adding an external time dependent bias $(V[s(\mathbf{x}, t)])$ to the Hamiltonian $\left(H_{0}(\mathbf{x}, t)\right)$ of the systems whose coordinates are denoted by $\mathbf{x}$, yielding a perturbed Hamiltonian $\left(H(\mathbf{x}, t)=H_{0}(\mathbf{x}, t)+V[s(\mathbf{x}, t)]\right)$, which does not correspond to the unbiased statistical mechanical ensemble in question (Tuckerman, 2010). In this work we use the scheme introduced by Hummer and Szabo (Hummer and Szabo, 2001):

$e^{-\beta G(s)}=\frac{\sum_{t} \frac{\left.\left\langle\delta[s-s(t)] e^{-\beta W_{t}}\right)\right\rangle}{\left\langle e^{-\beta W_{t}}\right\rangle}}{\sum_{t} \frac{e^{-\beta V(s, t)}}{\left\langle e^{\beta W_{t}}\right\rangle}}$,

where $\langle\ldots\rangle$ denote averaging over parallel realizations.

According to the second law of thermodynamics, it is only possible to provide an upper estimate of the equilibrium free energy related to a process from the work performed, and the equality between work and free energy holds only in the idealised case of transitions that occur at vanishing velocity. Equations based on Jarzinksy's equality (Gore et al., 2003) can infer free energy profiles from work profiles accompanying the transition that occurs at finite speed. Jarzinsky's equality ensures that while the individual trajectories, from which the work along the time dependent reaction coordinate $(s=s(t))$ is estimated, drive the system out of equilibrium, the free energy difference is calculated from ensemble averages over the microstates that describe a thermodynamic states along the path (Tuckerman, 2010). Thus instead of a single ideal thermodynamically reversible path, the free energy is estimated from a large number of irreversible pathways (that mimick the realistic uptake process) which are weighted according to their distance from the idealised path in the free energy representation. Jarzinsky's equality yields an estimate of the equilibrium free energy regardless of the velocity of the transition albeit based on a physically realistic set of sample processes.

In practice, Jarzinsky's equality and steered molecular dynamics have been successful at examining finite speed transitions between two states of a molecular system, e.g.: protein or nucleic acid unfolding (Park and Schulten, 2004; Gore et al., 2003). The timescale associated with a single water uptake event (impinging plus transport from the surface to the bulk) is $\sim 10 \mathrm{ns,}$ surface residence times are $\sim 1 \mathrm{~ns}$ (Bzdek and Reid, 2017). These timescales are matched by the length of individual realizations in our steered MD simulations. Additionally, initializing each realization (out-of-equilibrium simulation) from different starting configurations (Section 2.2) allows impact to happen at any point of the surface, thus incorporating thermodynamically suboptimal pathways - which have finite probability in experiments - are well represented in the sample. This approach confers an advantage for estimating the free energy profile compared to quasi-equilibrium approaches (i.e., umbrella sampling) in which the free energy represents the minimum energy pathway due to the time permitted for sampling the surface. In our systems, finite velocity pulling allows to sample transport through humps and wells of the corrugated intrinsic interface (Section 3.1.1). These corrugations represent thermodynamically different environments (Bartók-Pártay et al., 2008; Darvas et al., 2010a) for contact formation upon impact, and hence different transport pathways which can be relatively far away from the equilibrium one, but occur in real events with a finite probability. Note that exploring suboptimal pathways of interfacial mass transfer can be even more important in modeling transport through multicomponent surface films with heterogeneous lateral distributions. 
The steered MD method is illustrated in Figure 1. A sample steered MD trajectory is available as video supplement (https: //zenodo.org/record/4902870\#.YLsZwSORq_U).

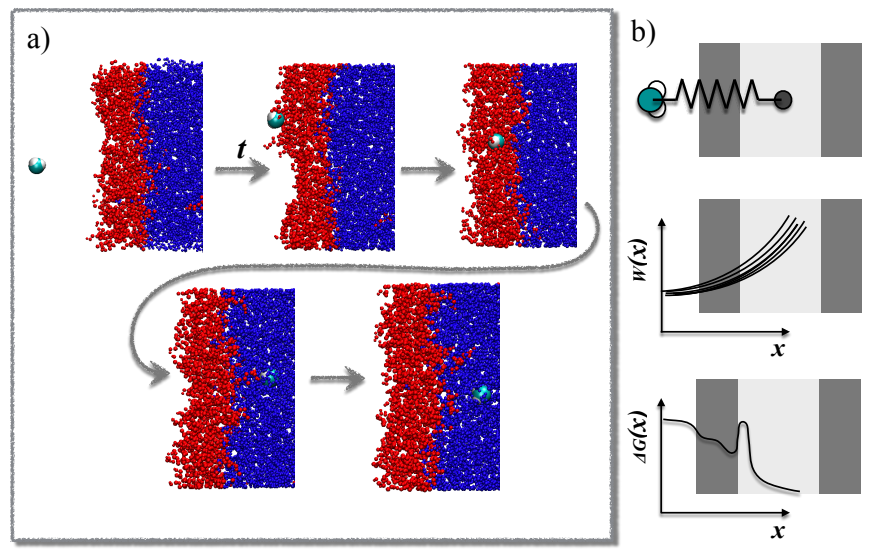

Figure 1. a) Example of the evolution of a steered MD simulation. b) Schematic summary of the free energy reconstruction protocol.

\subsection{Simulation details}

Steered MD simulations were performed using the GROMACS 5.1.3 program package (Abraham et al., 2015). The PLUMED 2.5 plugin (Tribello et al., 2014); was used to implement and control the steered MD simulations. The system consisted of a rectangular slab containing 5000 water molecules enclosed between two multilayers of hydroxy cis-pinonic acid (h-CPA), each of 125 molecules. The liquid slab was surrounded by a vapor phase from both sides (Figure 1). The average widths of the layers along the $\mathrm{Z}$ axis of the simulation box being $\sim 6.5 \mathrm{~nm}, \sim 2.5 \mathrm{~nm}$ and $\sim 6 \mathrm{~nm}$ for water, CPA and the vapor phase, respectively. The protocol to create and equilibrate such interfacial systems is described elsewhere (Hantal et al., 2010; Darvas et al., 2011b). A single water molecule is placed in the vapor phase of the preequilibrated interfacial system and pulled towards the middle of the aqueous phase along the reaction coordinate $(s(\mathbf{x}, t))$ - defined as the interface-normal $(\mathrm{Z})$ component of the distance connecting its center of mass to that of the aqueous phase by harmonic bias having a force constant of $\mathrm{k}=1000 \mathrm{~kJ}$ $\mathrm{mol}^{-1} \mathrm{~nm}^{-1}$. This condition satisfies the stiff spring approximation which enables the system to closely follow the path of the reaction coordinate by becoming the dominant force in the total dynamics Hummer and Szabo (2001).

100 parallel 6 ns-long realizations are performed at two temperatures, 200 and $300 \mathrm{~K}$ on the NVT (canonical) ensemble. The length of the simulation and the distance covered in the direction of the reaction coordinate results in an average pulling velocity of $1.2 \mathrm{~nm} \mathrm{~ns}^{-1}$. The realizations differ in the initial position of the gas phase water molecule in the X,Y plane parallel to the surface of the liquid slab, and in the random seed which is used to set initial velocity distributions. The choice of pulling velocity is a crucial point in setting up the simulation. It should be low enough to ensure that final and initial states represent the equilibrium distribution (Tuckerman, 2010), and also to yield numerically treatable forces. On the other hand, 
it has to be large enough to be drive the dynamics of the system, and finally too low transition velocities will undersample higher work pathways.Temperature is kept constant by means of the V-rescale thermostat (Bussi et al., 2007). Water molecules are described by the TIP4P water model (Jorgensen et al., 1983) and h-CPA molecules by the OPLS potential (Jorgensen and Tirado-Rives, 1988). Alkyl groups are treated as united atoms, while other hydrogens are treated explicitly. Long range electrostatics are accounted for by the particle mesh Ewald method (Essmann et al., 1995) beyond a cutoff of $1 \mathrm{~nm}$, while Lennard-Jones interactions are smoothly truncated to zero beyond the same cutoff.

\section{Results and discussion}

Free energy profiles are interpreted in terms of the local characteristics of the simulated systems and a detailed mechanism of water uptake is proposed. The decomposition of the free energy profiles into enthalpic and entropic contributions allows us to identify the thermodynamic drivers of interfacial mass transfer of water. The effect of the complex water uptake mechanism on particle growth and activation is then assessed by estimating interfacial transfer coefficients. Implications for droplet formation are then discussed in the light of partitioning timescale estimates, intraparticle distribution of water within the organic shell, and equilibrium saturation ratios.

\subsection{Structural and thermodynamic characteristics}

\subsubsection{Intrinsic density profiles}

Local nanoscale fluctuations of fluid interfaces from capillary waves create surface corrugations (Rowlinson, 1982) whose amplitude varies between 0.3 and $1 \mathrm{~nm}$ depending on the chemical composition of the surface (Hantal et al., 2010; Darvas et al., 2011a). These corrugations do not average out during typical timescales of interfacial mass transfer of a water molecule and are enhanced when a molecule crosses the interface (Benjamin, 1993; Karnes and Benjamin, 2016; Benjamin, 2019). Thermal fluctuations are not accounted for by traditional surface definitions and cause artificial smoothing of the surface and systematic errors in the selection of surfaces molecules (Bartók-Pártay et al., 2008; Jorge et al., 2010). Intrinsic surface analysis - i.e. decoupling surface fluctuations from the determining interfacial properties (Appendix A) - of molecular simulation trajectories is necessary to resolve the effect of capillary waves on density (Jorge et al., 2010) and free energy profiles (Darvas et al., 2013; Braga et al., 2016; Klug et al., 2018) results are sharper and contain features which are smoothed out by fluctuations using traditional surface definitions. In this work, intrinsic analysis is limited to the selection of surface molecules and the calculation of density profiles anchored to the fluctuating surface by the ITIM algorithm (Sega et al., 2018), which allows to establish qualitative connections between the free energy profiles and the local perturbations of solution structure and thermodynamic properties in the vicinity of interfaces. On the other hand, finite velocity trajectories ensure that fluctuations are not averaged out during a single realisation, allowing for modeling thermodynamically different pathways resulting from surface corrugations.

Shaded blue and yellow areas in Figure 2 a) and b) show the intrinsic mass density profiles of the aqueous and the organic phase. Organic density profiles reveal that the thickness of the organic phase is $\sim 1.8$ and $\sim 2.1 \mathrm{~nm}$ at 200 and $300 \mathrm{~K}$, respec- 
tively, measured at $5 \%$ of the average height of the profile. It is sufficient to accommodate a disordered multilayer, but is too low to allow the formation of a bulk phase. The lack of a bulk phase is evidenced by the two neighboring peaks - characteristic of interfaces - in the density profile of the organic phase with no plateau at the bulk phase density $\left(1.2 \mathrm{~g} \mathrm{~cm}^{-3}\right)$ in between. A $215 \sim 85 \%$ local drop in the total density (sum of water an organic density) compared to that of bulk aqueous phase can be observed at the Gibbs dividing surface of the two condensed phases at both temperatures. The affected region is wider at $300 \mathrm{~K}$. The aqueous phase has two consecutive peaks characteristic of the first two molecular layers, followed by a bulk phase plateau. The interface and subsurface structure are similar, but more pronounced at $200 \mathrm{~K}$ as result of reduced thermal motion which preserves layered structure at low temperatures. The interfacial region is characterized by an overlap between the organic and the water density profiles which is due to partial miscibility of the two phases. The width of this mixed region is $1.5 \mathrm{~nm}$ at 300 $\mathrm{K}$ and $1.2 \mathrm{~nm}$ at $200 \mathrm{~K}$ disregarding the small peak of organics in the bulk aqueous phase.

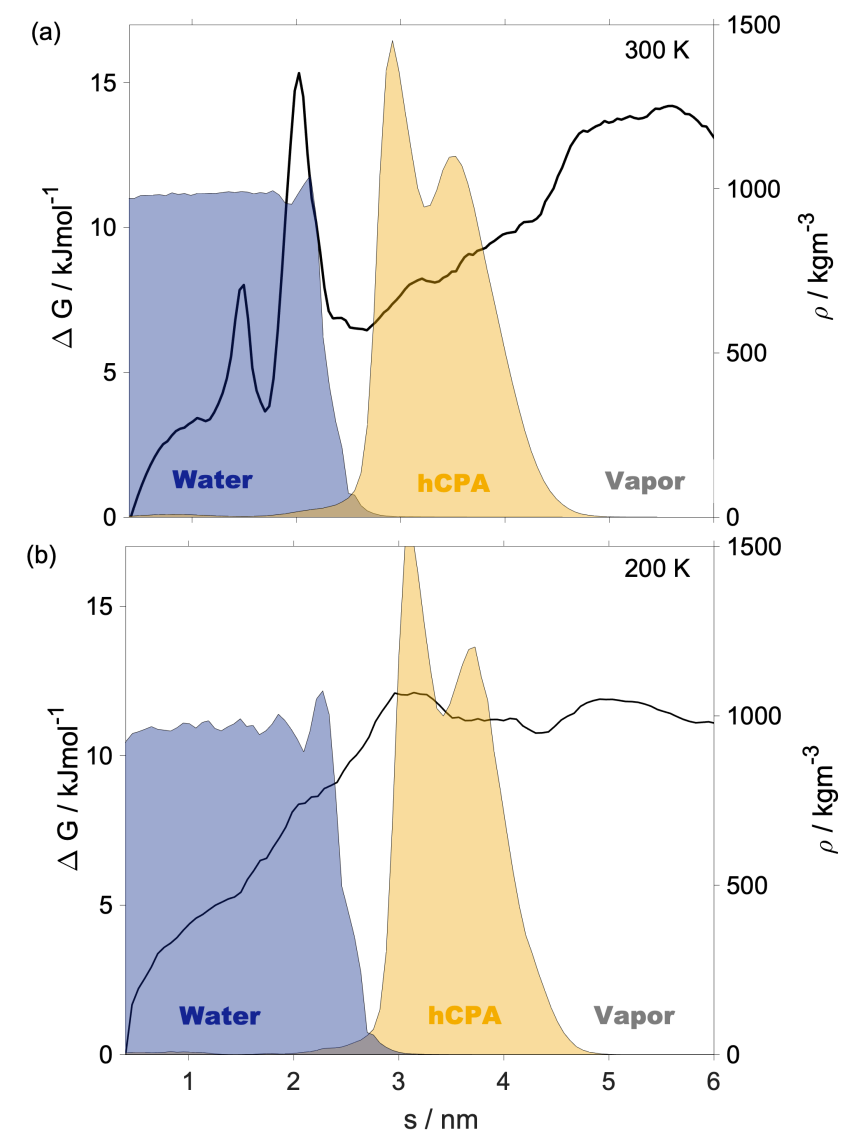

Figure 2. a) Free energy and density profiles at $T=300 \mathrm{~K}$; b) the same profiles at $\mathrm{T}=200 \mathrm{~K}$ 


\subsubsection{Free energy profiles}

Helmholtz free energy profiles, which represent the thermodynamic state function on the canonical ensemble. While the Helmholtz free energy is formally different from the experimentally determined Gibbs free energy, in systems containing incompressible condensed phases the additional $p \Delta V$ term is negligibly small; thus the two quantities can be assumed to be the same within a small error. Free energy profiles with the reference state assigned to the bulk aqueous phase are shown as black solid lines in Figure 2 a) and b). Profiles are significantly different at the two temperatures which suggests that mechanism of gas-to-particle partitioning in LLPS particles is temperature dependent. Despite differences, simulations show two common characteristics: i) the negative free energy difference $\left(\sim-14 \mathrm{~kJ} \mathrm{~mol}^{-1}\right.$ at $300 \mathrm{~K}$ and $\sim-11 \mathrm{~kJ} \mathrm{~mol}^{-1}$ at $\left.200 \mathrm{~K}\right)$ between the bulk aqueous and the vapor phase, which underlines that overall water uptake from the vapor phase is thermodynamically favored, and, ii) the lack of a free energy barrier at the vapor/organic interface, which is the main difference between free energy profiles of transfer through hydrophobic media, for which large maxima have been observed leading to reduced surface accommodation coefficients (Sakaguchi and Morita, 2012; Ergin and Takahama, 2016).

The free energy profile at $300 \mathrm{~K}$ begins with a plateau characteristic of the vapor phase, followed by a steep decrease $(\sim 5$ $\mathrm{kJ} \mathrm{mol}^{-1}$ ) at the vapor/organic interface. A monotonic decrease in the free energy profile characterizes the transfer of the water molecule in the organic phase. The lack of a plateau is related to the fact that the organic layer is not thick enough to accommodate a bulk phase, which results in a position dependent anisotropy caused by the proximity of both interfaces in combination with the partial dissolution of the water in the organic phase, evidenced by non-zero water density up to $s \approx 3.5 \mathrm{~nm}$. The presence of dissolved water molecules results in the increased slope of the profile near the water/organic interface. The free energy profile has a local minimum at the organic/water interface, and a global maximum $\left(\sim 15 \mathrm{~kJ} \mathrm{~mol}^{-1}\right)$ corresponding to the first molecular layer of water, seen as a peak in the intrinsic density profile. A second peak and a smoothly decreasing region follow. The free energy profile at $200 \mathrm{~K}$ is considerably smoother; the magnitude of none of the features exceeds the energy of thermal motion $\left(3 / 2 k_{B} T\right)$ at the corresponding temperature, thus any small local minimum or maximum can be viewed as statistically insignificant. The minimum and the peak at the organic/water interface characteristic of the room temperature profile cannot be observed at low temperature. While vapor-to-organic transfer is a favorable and barrierless transition at both temperatures, the transport between the organic shell and the core is hindered by the presence of the free energy barrier at 300 $\mathrm{K}$, which diminishes at low temperature. Large temperature induced differences suggest that the thermodynamic driving forces are strongly temperature dependent. One possible explanation is that they are of entropic natrure, and their strength is explicitly scaled by the temperature through the $-T \Delta S$ term.

\subsubsection{Internal energy and entropy profiles}

To understand the thermodynamic drivers of water uptake, free energy profiles are decomposed into entropy and internal energy profiles shown in Figure 3. The sum of the interaction energies of the pulled water with the surrounding molecules is used as a surrogate for the internal energy, the calculation is described in details in Appendix B. The total entropy profile is obtained by subtracting the internal energy profile from the free energy profile, and some distinct contributions to the total entropy are 
calculated. The entropy contributions that are analysed in detail are not necessarily additive or complete, but selected to explain the features of the free energy profiles.
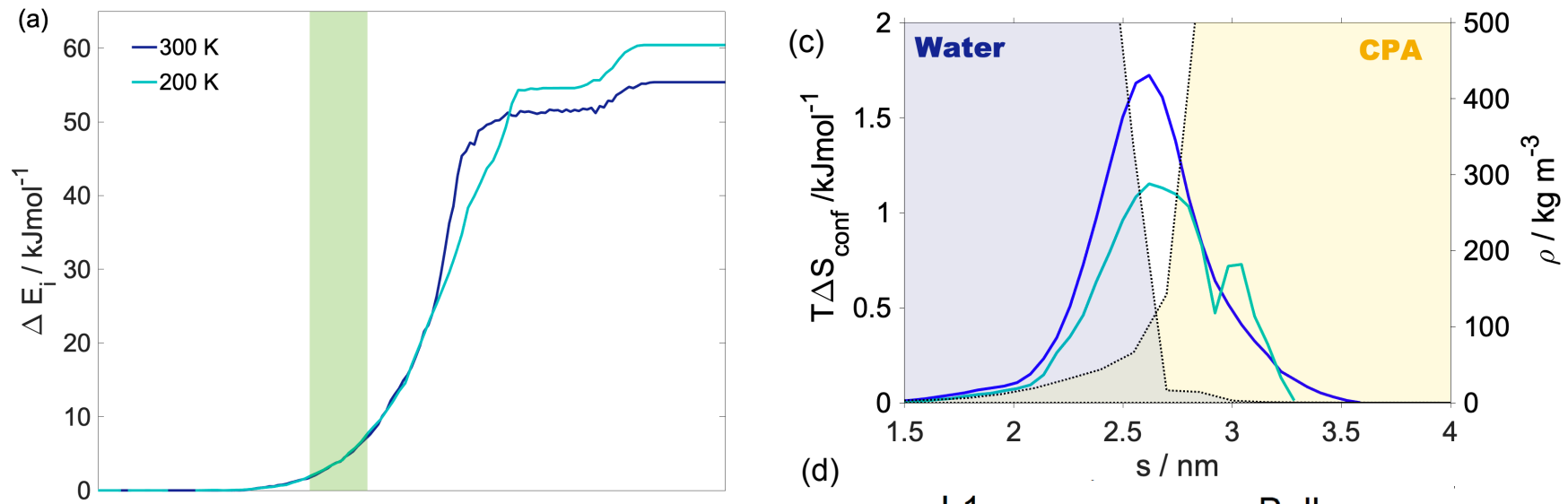

(d) $\mathrm{s} / \mathrm{nm}$
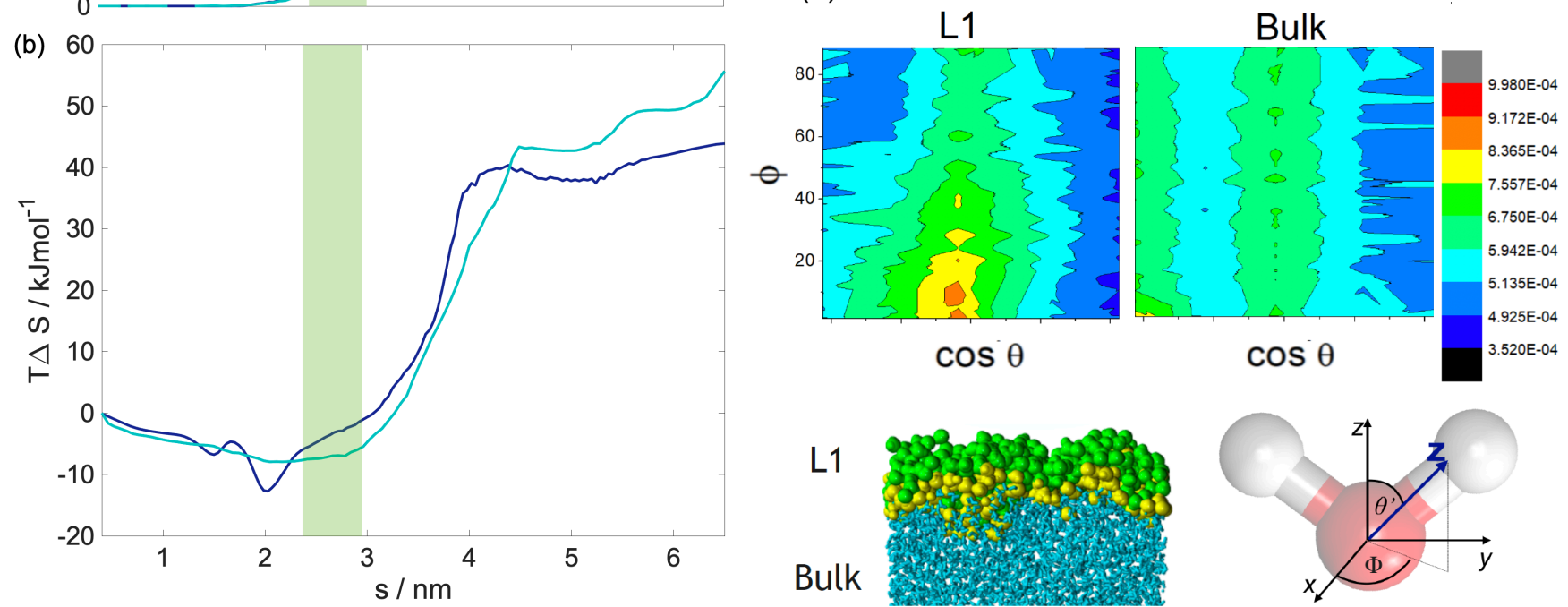

Figure 3. a) Internal energy, b) entropy profiles as a function of the reaction coordinate used in the steered MD simulations, the green shaded area denotes the interfacial region between the organic and aqueous phase c) The conformational entropy in the interfacial region overlapped with intrinsic density profiles of the aqueous (blue shaded) and the organic (yellow shaded) phase d) Top panel: Orientational maps of the water molecules in the first molecular layer (L1) of the aqueous phase and in a randomly selected layer in the bulk phase. Bottom panel left: An equilibrium snapshot from a simulation showing the first two molecular layers (green and yellow balls) and the bulk phase (cyan sticks) as determined by the ITIM algorithm. Bottom panel right: the definition of the orientational vectors of the water molecule.

Internal energy profiles in Figure 3 a) show that water uptake is energetically favorable, the difference between the pulled water molecule being in the vapor phase and in the aqueous phase is $\sim-55 \mathrm{~kJ} \mathrm{~mol}^{-1}$ at $300 \mathrm{~K}$ and $\sim-60 \mathrm{~kJ} \mathrm{~mol}^{-1}$ at 200 $\mathrm{K}$. This corresponds approximately to the formation of 3-4 hydrogen bonds by the pulled molecule if the average energy of a hydrogen bond is assumed to be $15-20 \mathrm{~kJ} \mathrm{~mol}^{-1}$ (Wendler et al., 2010), which is also supported by the hydrogen bond profile 
calculated for a randomly selected realization (Appendix A). Internal energy profiles are smooth and show a similar overall behavior at the two temperatures. A small drop in the internal energy whose magnitude is $\sim 3 \mathrm{~kJ} \mathrm{~mol}^{-1}$ and $\sim 5 \mathrm{~kJ} \mathrm{~mol}^{-1}$ at the 200 and $300 \mathrm{~K}$ respectively indicates the formation of contact with the organic phase. It is however not sufficiently low compared to the internal energy observed in the subsequent bulk organic phase to energetically stabilise a surface adsorbed state. Both internal energy profiles have a plateau spanning the outer half of the organic phase, where water molecules from the bulk aqueous phase do not penetrate. This is followed by a smoothly decreasing part which corresponds to the pulled molecule forming an increasing number of hydrogen bonds with both h-CPA and the water molecules dissolved in the organic phase. Near the organic-water interface and in the aqueous phase the internal energy profiles are close to identical at the two temperature, suggesting that specific features of the free energy profile are of entropic origin.

Total entropy profiles (Figure $3 \mathrm{~b}$ ) bear all the features that appear in the free energy profiles and their shape is different at the two temperatures. In the followings these profiles are further decomposed into specific terms: configurational, interfacial, conformational and orientational entropy. Configurational entropy profiles calculated using Schlitter's formula (Baron et al., 2006) as explained in Appendix B are close to constant throughout the condensed phase, $-T \Delta S_{\text {config }}=25 \mathrm{~kJ} \mathrm{~mol}^{-1}$ at $300 \mathrm{~K}$ and $14 \mathrm{~kJ} \mathrm{~mol}^{-1}$ at $200 \mathrm{~K}$. They are thus not responsible for any of the features in the free energy profiles. Interfacial entropy $\left(T \Delta S_{I F}\right)$ at the vapor/organic interface is approximately $5 \mathrm{~kJ} \mathrm{~mol}^{-1}$ at $300 \mathrm{~K}$ and is negligibly small at $200 \mathrm{~K}$ (Appendix B). Despite of the positive value of interfacial entropy the overall entropy difference between the vapor and the organic phase is a small negative value, which — together with the moderate change in enthalpy at the interface — is responsible for the lack of surface adsorbed states. A possible explanation for the overall negative entropy is the ordering of the organic molecules at the surface which reduces orientational degrees of freedom in a similar manner as described later for the water/organic interface.

Conformational entropy profiles, calculated from the mole fraction profiles of the water and the organic molecules (Appendix B), are shown in Figure $3 \mathrm{c}$ ). They exhibit a peak located at the organic/water interface at both temperatures and are close to zero elsewhere. These peaks represent the effect of non-ideal local mixing of two phases in this region. The conformational entropy peak is slightly higher at $300 \mathrm{~K}$, mostly due to the higher temperature. This and the local $\sim 85 \%$ decrease in the total density in this region explain the presence of the free energy minimum at the organic/water interface. Lower density ensures the reduction of steric hindrance for any conformations without loosing hydrogen bonds. These explain the minimum in the free energy profile observed at the water/organic interface at $300 \mathrm{~K}$. High conformational degrees of freedom manifest in subsequent detaching/attaching of the pulled molecule between the organic and aqueous phase in varying orientations, which can be observed only in the $300 \mathrm{~K}$ simulations (video supplement available). The conformational entropy profile peak in the interfacial region is significantly $(\sim 40 \%)$ smaller at $200 \mathrm{~K}$. Additionally, the low density region is narrower due to the pronounced shoulder in the water density profile (Figure 2), thus steric effects are not as effectively reduced as at 300 $\mathrm{K}$. The joint decrease of the magnitude of both contributions leads to the disappearance of the free energy minimum at the organic/water interface at $200 \mathrm{~K}$.

Increased orientational order of molecules at interfaces of molecular liquids locally reduces orientational entropy, which is responsible for the free energy peak coinciding with the first two molecular layers of water in the $300 \mathrm{~K}$ simulations. Interfacial molecules adapt preferred orientations owing to topological anisotropy of intermolecular interactions, in contrast to the bulk 
where the close to isotropic energetic environment ensures nearly random orientations. To illustrate the differences between the orientational preferences of interfacial and bulk water molecules we calculate the joint distribution of the two angles defined in the bottom right panel of Figure $3 \mathrm{~d}$ ) in the first molecular layer and in a randomly selected molecular layer from the bulk aqueous phase. Details of the calculation are summarized in Appendix B. The two dimensional joint distribution of these two angles (Figure $3 \mathrm{~d}$ ), top panel) is able to define the orientation of a rigid body having a $C_{2 v}$ point group symmetry with respect to an external axis. The peak shows that in the first layer water molecules tend to lie parallel to the surface, whereas the bulk phase distribution shows no significant preferences. Increased orientational order results in a decrease in orientational entropy, corresponding to the maximum of the free energy profile. The second layer of water has similar orientational preferences (Appendix B), which explains the subsequent smaller free energy peak. Similar results are found for $200 \mathrm{~K}$ (Appendix B), thus the observed temperature dependence results again from the explicit scaling of the importance of entropy with temperature. Orientational entropies are also estimated in 1 dimension (Appendix B), but due to symmetry reasons they do not give a quantitative description of the ensemble of ordering effects.

\subsubsection{Generalization of the driving forces}

The question whether the thermodynamic driving forces identified in the previous section are system specific or generalizable over a wider range of compositions that can yield LLPS particles is important for determining the level of confidence with which implications to atmospheric aerosol can be stated based on these simulations. The most prominent characteristics of the free energy profiles are i) the lack of a minimum at the vapor/organic interface, ii) the minimum at the organic/water interface and iii) the maximum corresponding to the first layer of the aqueous phase.

Experimental and molecular simulation studies show that driving forces which lead to the appearance of these features are generally present in interfacial systems involving molecular liquids or solids. i) The value of the free energy at the vapor/organic interface is moderated by the increased order of organic molecules which has been observed from experiments and simulations in pure water (Cipcigan et al., 2015), pure organics (Darvas et al., 2010a) as well as in concentrated (Darvas et al., 2010b) and dilute aqueous organic solutions (Takamuku et al., 1998; Pártay et al., 2008; Pojják et al., 2010; Ghatee et al., 2011; Makowski et al., 2016). ii) The density drop in the interfacial region and the enhanced local mixing which invoke the free energy minimum at the organic/water interface at $300 \mathrm{~K}$ are characteristic of any liquid/liquid interface (Hantal et al., 2010; Jorge et al., 2010; Darvas et al., 2011b, 2013). The extent of local mixing is trivially determined by the hydrophilicity of the organic compound. iii) Orientational preferences of water molecules near liquid/liquid or liquid/solid interfaces, which account for the maximum of the free energy profiles are also generally present in interfacial systems. They have been observed in molecular simulations several times at various liquid/liquid interfaces such as carbon tetrachloride/water (Hantal et al., 2010; Kertész et al., 2014) or dichloroethane/water (Hantal et al., 2010) and proved to be enhanced next to a solid counter phase (Kertész et al., 2014).

The strong temperature dependence follows directly from the entropic nature of these driving forces and the definition of the free energy, consequently besides the presence of the driving forces, their temperature dependence is also expected to be generally valid. LLPS is known to form if the $\mathrm{O}: \mathrm{C}<0.8$, the organic components in the examples listed above cover the complete $\mathrm{O}: \mathrm{C}$ ratio range. Thus our findings are likely not system specific, and represent a typical behavior for sparingly soluble organic 
compounds present in the atmosphere, although exact values of the thermodynamic quantities can vary from system to system. The following implications are thus quantitatively only valid for the water/h-CPA/vapor system, while qualitatively similar behavior can be expected for a wider variety of LLPS forming cases.

\subsection{Implications for water uptake and particle growth kinetics}

\subsubsection{Interfacial transfer coefficients}

Interfacial transfer coefficients are estimated from the activation free energies characteristic of the vapor-to-organic $\left(k_{v / o}\right)$, organic-to-water $\left(k_{o / w}\right)$ and vapor-to-water $\left(k_{v / w}\right)$ transfer using the transition state theory as:

$k_{i / j}=\exp \left[\frac{-\Delta_{i j}^{\#} G}{R T}\right]$,

where $i$ and $j$ indicate the phases forming the interface in question. The corresponding activation free energies are illustrated using the $300 \mathrm{~K}$ free energy profile in Figure $4 \mathrm{a}$ ). While the transfer coefficient calculated here is conceptually different from the mass accommodation coefficient used in atmospheric applications, chemical kinetic frameworks are widely used to estimate $\alpha$ from free energy profiles (Grote and Hynes, 1980; Taylor and Garrett, 1999; Truhlar and Garrett, 2000; Sakaguchi and Morita, 2012; Ergin and Takahama, 2016). We note that due to the lack of a well-defined bulk phase plateau in the organic phase we choose an average free energy characteristic of the middle of the organic phase as a reference value. This choice is arbitrary and a different definition may slightly modify obtained transfer coefficients, albeit without significantly altering values, trends and conclusions. Vapor-to-organic (surface) transfer coefficient $\left(k_{v / o}\right)$ are near unity at both temperatures, whereas organicto-water (core) transport is characterized by $k_{o / w}=0.05$ at $300 \mathrm{~K}$, and $k_{o / w}=1$ at $200 \mathrm{~K}$. To complete the analysis, we also estimate a hypothetical transfer coefficient of the uptake of water from the vapor phase by the aqueous core disregarding the organic phase, $k_{v / w}$ which is equal to 0.38 at $300 \mathrm{~K}$ and unity at $200 \mathrm{~K}$.

The vapor-to-organic transfer coefficients resemble most closely the commonly used definition of the mass accommodation coefficient, which considers either adsorption at the surface or absorption in the first few molecular layers of the particle phase as the final state of gas-to-particle partitioning. Due to the lack of maxima or minima at the vapor/oragnic interface, in our systems surface adsorbed states of the water are thermodynamically indistinguishable from those with the water absorbed by the subsurface region. 
(a)
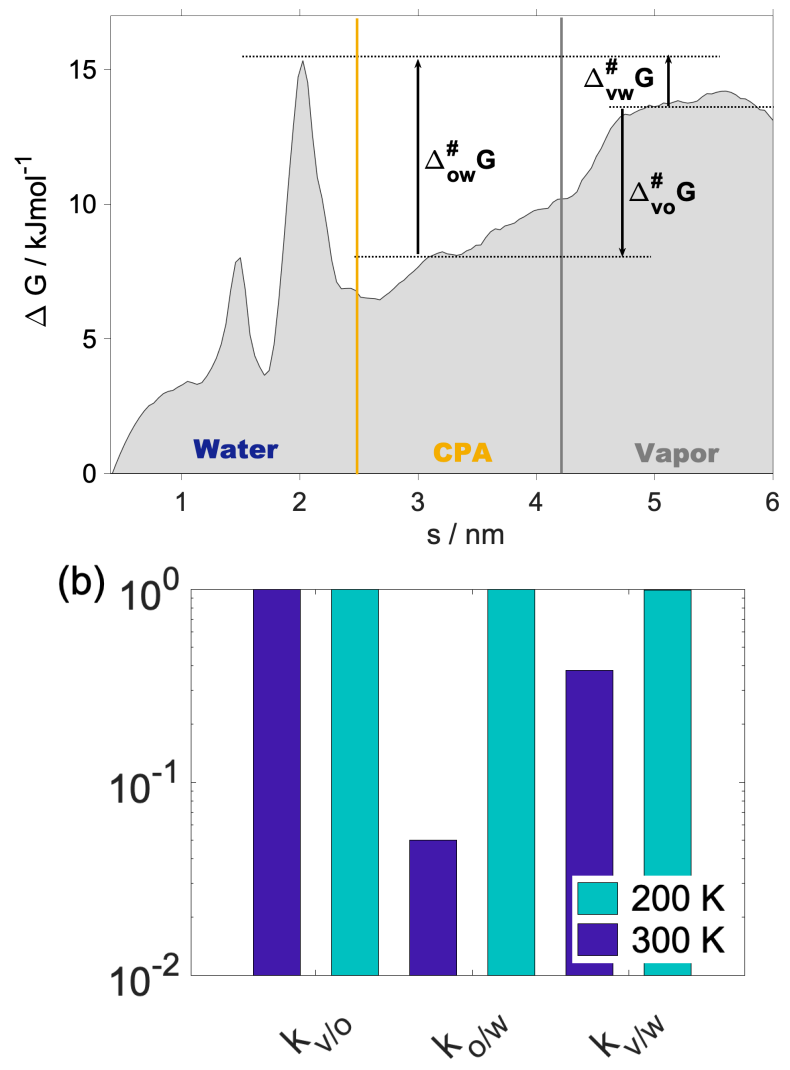

Figure 4. a) Definition of activation free energies at the different interfaces at $T=300 \mathrm{~K}$. b) Transfer coefficients at the different interfaces dark blue $300 \mathrm{~K}$, light blue $200 \mathrm{~K}$

The organic-to-water $\left(k_{o / w}\right)$ and vapor-to-water transfer coefficients $\left(k_{v / w}\right)$ are reduced compared to the mass accommodation coefficients $\left(k_{v / o}\right)$ at room temperature owing to the free energy barrier corresponding to the first molecular layer of water. As opposed to mass accommodation coefficients which show no temperature dependence, $k_{o / w}$ and $k_{v / w}$ follows a similar trend with temperature as that observed for water uptake on a hexadecanol monolayer (Davies et al., 2013), and on pure water (Davidovits et al., 2004). $k_{o / w}$ describes the lower bound of the probability of a gas phase water molecule being absorbed by the aqueous core of the phase separated particle. $k_{v / w}$ represents an upper bound of the same probability. The two values thus define the range of core uptake coefficients. The fact that core uptake coefficients differ significantly from mass accommodation coefficients highlights the possibility that the traditional representation of water uptake by a single value of $\alpha$ or an effective uptake coefficient may have to be complemented by a temperature-dependent core uptake term to fully describe water uptake by phase-separated aerosol. 


\subsubsection{Mass accommodation coefficients}

Mass accommodation coefficients defined by vapor-to-organic transfer coefficients are near unity, and agree well with the globally representative values proposed in modeling studies (Raatikainen et al., 2013), as well as with recent experiments which report large mass accommodation coefficients (Liu et al., 2019) and unhindered gas-to-particle partitioning of water and organics in phase separated particles (Gorkowski et al., 2017). While global datasets of CCN concentrations can usually be described by $0.1<\alpha<1$, implying uninhibited water uptake (Raatikainen et al., 2013), compressed hydrophobic organic films can result in mass accommodation coefficients as low as 0.001 . In such systems, $\alpha$ is correlated with the integrated carbon number density (Ergin and Takahama, 2016) of the organic layer, estimated as the integral of the density profile along the outer half of the organic layer divided by its width. The integrated carbon number density for our systems is $14 \mathrm{~nm}^{-3} \mathrm{which}$ for a completely hydrophobic film comprised solely of aliphatic $\mathrm{CH}$ moieties would correspond to $\alpha \sim 0.01$ (Ergin and Takahama, 2016) instead of the observed near unity values. In the presence of hydrophilic functional groups - which form hydrogen bonds and dipole-dipole interactions — such a simple descriptor is insufficient to reliably predict mass-accommodation coefficients.

Reduction in mass accommodation coefficients result in increased CCN number concentrations. The explanation for this effect is that droplet number concentrations in ambient clouds depend on the maximum supersaturation, $S_{\text {max }}$, which is largely determined by the condensation rate of water vapor on the growing droplets. Reduced $\alpha$ values correspond to low water vapor condensation rates - which allow supersaturation to develop for longer periods of time (compared to when $\alpha$ is larger), resulting in increased $S_{\max }$ and droplet concentrations. Models simulations predict a mild 1.2-fold increase in CCN number concetrations for $\alpha=0.1$, a 1.5-1.8-fold increase for $\alpha=0.01$ and 2-2.5 fold increase for $\alpha=0.001$ (Raatikainen et al., 2013). In this framework, near-unity mass accommodation coefficients obtained from our simulations at both temperatures are not expected to alter droplet number concentrations, further supporting that kinetic delays do not explain the increased CCN activity of LLPS aerosol.

\subsubsection{Core uptake coefficients}

Hindered mass transfer of water between the organic shell and aqueous core together with uninhibited mass accommodation of water in LLPS aerosol results in different condensational growth rates of the core and shell, leading to a dynamic retention of water by the organic shell. We use the $k_{v / o} / k_{o} / w$ ratio to estimate the extent of dynamic retention of water by the organic phase. The value is approximately unity at $200 \mathrm{~K}$, and about 20 at $300 \mathrm{~K}$. The growth rate of the organic shell is thus substantially larger at $300 \mathrm{~K}$ than that of the core, which in agreement with results of multilayer kinetic model (KM-GAP) calculations which also evidence faster condensational growth of the particle shell than of core (Shiraiwa et al., 2013). This suggests that the aqueous core contains less water and the organic shell is more dilute at any time during growth of the particle than predicted assuming that mass transfer kinetics can be described by a single $\alpha$.

Retention of water in the organic shell due to reduced core uptake coefficient (organic-to-water or vapor-to-water transfer coefficient) may affect equilibrium properties (vapor pressure and surface tension) which determine cloud droplet growth and activation. Increased water content of the organic shell can increase vapor pressure and surface tension, which both affect cloud 
droplet growth and activation. In the extreme case, inhibited transport between the shell and the core, indicated by reduced core uptake coefficients, may invoke swelling and - depending of the solubility of the organic compounds — dissolution of the shell. These hypotheses can be relevant if the vapor phase is in dynamic equilibrium with the organic shell containing an increased amount of water and this equilibrium is unaffected by the presence of the aqueous core, in other words water uptake happens only by the shell. For this condition to hold gas-to-particle partitioning timescale of water $\left(\tau_{s}\right)$ should be significantly shorter than timescale of transfer from the shell to the core $\left(\tau_{c}\right)$. Timescales are estimated as the e-folding times of condensation-evaporation, assuming that both gas-to-particle and shell-to-core partitioning can be described as (Saleh et al., 2013):

$405 \tau=\frac{1}{2 \pi D F d N}$,

where $F=(1+K n) /(1+0.3773 K n+1.33 K n(1+K n) / \alpha)$ is the Fuchs-Sutugin correction factor, with $K n$ being the Knudsen number and $\alpha$ the transfer coefficient of the given process. For gas-to-particle partitioning $K n=10^{-2}, 10^{-1}, 10^{0}, \alpha=1$, the gas phase diffusion coefficient of water is estimated at $0.26 \mathrm{~cm}^{2} \mathrm{~s}^{-1}$ and $0.128 \mathrm{~cm}^{2} \mathrm{~s}^{-1}$ at 300 and $200 \mathrm{~K}$ (Pruppacher and Klett, 2010), while $d$ is the diameter of the particle $(100 \mathrm{~nm})$. For core-to-shell partitioning $K n$ is approximated as the ratio of the diameter of the h-CPA molecules and the width of the organic layer, $\alpha=k_{o / w}$ in one set of calculations and $\alpha=k_{v / w}$ in another set, $d$ is the core diameter, and the diffusion coefficient $(D)$ is varied between $10^{-3}$ and $10^{-8} \mathrm{~cm}^{2} \mathrm{~s}^{-1}$. The ratio between the core and the full particle diameter is the same as the ratio of the width of the organic shell and the total system width. Assuming the particle number concentration $(N)$ to be $1000 \mathrm{~cm}^{-3}$, the equilibration timescale of gas-to-particle partitioning $\tau_{s}$ is on the order of 1-2 minutes, similar to timescales reported for the equilibration of semivolatile molecules(Saleh et al.,

415 2013). $\tau_{c} / \tau_{s}>10^{3}$ in every case (Figure 5); thus the hypothesis of equilibrium between the organic shell and the vapor phase holds for our system at both temperatures and the water uptake by the particle core is somewhat hindered. The ratio of the timescales is at least three orders of magnitude even when the core and shell uptake coefficients are assumed to be both unity $(200 \mathrm{~K})$, owing to the difference between gas and particle phase diffusivity. Reduced core uptake coefficients further increase this difference by orders of magnitude depending on whether the upper $\left(k_{v / w}\right)$ or the lower $\left(k_{o / w}\right)$ estimate of the core uptake coefficient is used. In summary, as expected the equilibration timescale of gas-to-particle partitioning is sufficiently short to assume equilibrium between the vapor phase and the organic shell, while core uptake may not reach equilibrium within a typical model of cloud updraft. 


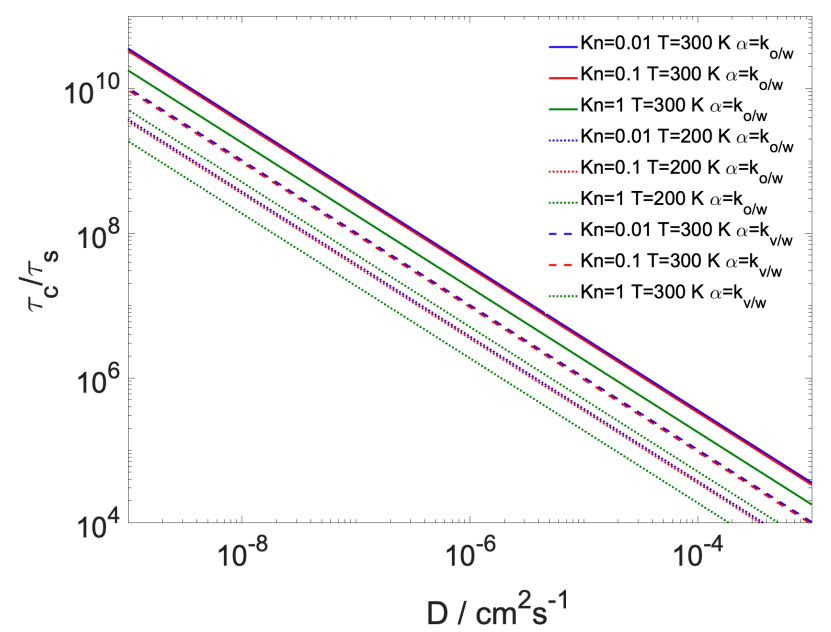

Figure 5. The ratio of characteristic timescales of shell-to-core and gas-to-particle partitioning as function of bulk phase diffusivity of the water in the organic shell at varying gas phase Knudsen numbers and temperature. At $300 \mathrm{~K}$ model calculations are presented using both the lower and the upper of the core uptake coefficient.

\subsubsection{The effect of bulk diffusion and non-uniform concentration distribution}

The non-negligible free energy difference between the vapor/organic and the organic/water interface potentially alters the above described uniform increase of the water concentration in the shell and carries consequences for droplet growth and activation. A more detailed understanding of these effects can be obtained by converting the free energy profile using the expression $\Delta G(s)=-k_{B} T \ln c_{\text {rel }}(s)$ into a probabilistic density profile $c_{\text {rel }}(s)$, which corresponds to an equilibrium concentration profile of the condensing water in the organic phase at arbitrary values of the vapor pressure. This equilibrium concentration profile is calculated under the assumption of instantaneous diffusion and bears no information about the surrounding relative humidity $(\mathrm{RH})$, and is valid for a mostly-organic shell (as the free energy profiles were derived for pulling simulations of single water molecules). The effect of non-instantaneous bulk phase diffusion of water and RH are accounted for by a correction factor, $f$ :

$c_{\text {rel }}(s)=\exp \left[\frac{-\Delta G(s)}{k_{B} T}\right] f\left(D_{p}, C_{v}\right) ; f\left(D_{p}, C_{v}\right)=\frac{1}{1+\frac{\alpha \omega C_{v}}{4 D_{p} \rho_{p}}}$.

$C_{v}$ and $D_{p}$ are the vapor phase concentration of water and the bulk phase diffusion coefficient of water in the organic phase, respectively, $\omega$ is the mean thermal velocity of water in the vapor phase, $\alpha$ is the mass accommodation coefficient, and $\rho_{p}$ is the density of the organic phase $\left(1.2 \mathrm{~g} \mathrm{~cm}^{-3}\right.$ in our calculation). The correction factor was adapted from Shiraiwa and Pöschl (2020), who derived an expression to account for the effect of diffusivity on gas-to-particle partitioning by introducing a penetration-depth-dependent definition of the mass accommodation coefficients of organic molecules absorbed by aerosol particles used in particle growth kinetic models (Shiraiwa et al., 2012). Our model calculations are performed at five RH values in the range of 50-98\%; using 13 different diffusion coefficients between $10^{-15}$ and $10^{-3} \mathrm{~cm}^{2} \mathrm{~s}^{-1}$, characteristic of liquid and semisolid particles. Given that the glass transition temperature of pure h-CPA is around $200 \mathrm{~K}$ (Shiraiwa et al., 2017), we 
expect the organic shell to be in the liquid state at $300 \mathrm{~K}$, while both phase states are possible at $200 \mathrm{~K}$. $c_{\mathrm{rel}}(s)$ is normalized to the $0-1$ range, with 0 and 1 corresponding to the minimum and maximum of the $c_{\text {rel }}(s)$ profile within the organic phase at 300 $\mathrm{K}$.
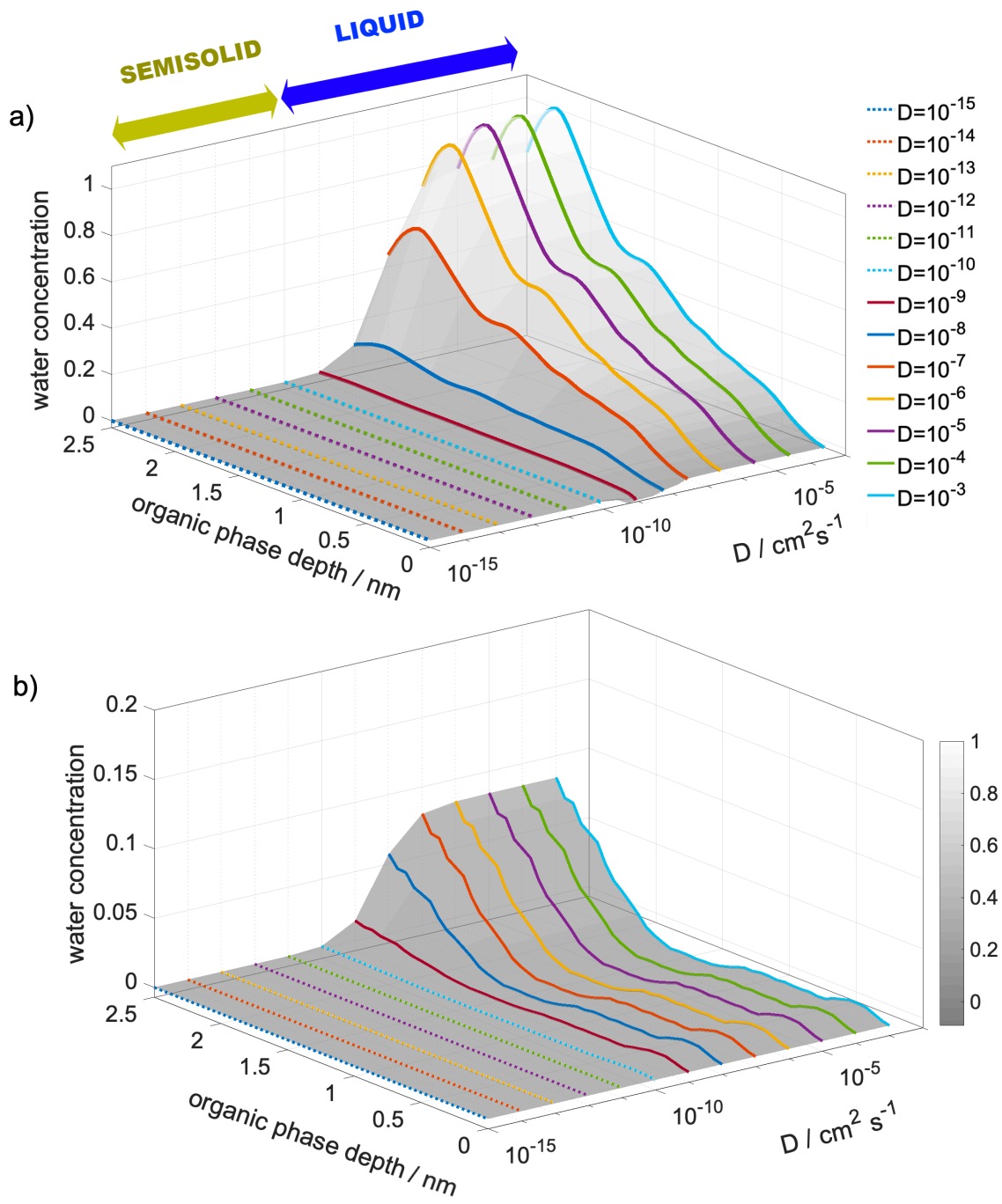

Figure 6. Modeled, diffusivity-corrected concentration gradient of water in the organic phase at a) $300 \mathrm{~K}$ and b) $200 \mathrm{~K}$ at $98 \% \mathrm{RH}$ and at varying diffusion coefficients. Organic phase depth describes the distance from the vapor/organic interface, zero corresponds the Gibbs dividing surface between the vapor and particle phase 
Figure 6 shows the equilibrium diffusion-corrected concentration profiles at RH=95\% at $300 \mathrm{~K}$ (a) and at $200 \mathrm{~K}$ (b). Condensing water molecules show a strong preference to be accommodated near the organic/water interface, while the vicinity of the vapor/organic interface is depleted in water at $300 \mathrm{~K}$ (Figure 6) a)) for bulk phase diffusion coefficient values characteristic of the liquid phase $\left(10^{-3}<D_{p}<10^{-9} \mathrm{~cm}^{2} \mathrm{~s}^{-1}\right)$. A much less pronounced concentration gradient, about an order of magnitude smaller than that found at room temperature, can be observed at $200 \mathrm{~K}$ if liquid phase diffusivities are assumed. The effect of diffusion is negligible in the $10^{-3}<D_{p}<10^{-6} \mathrm{~cm}^{2} \mathrm{~s}^{-1}$ diffusivity range. The steepness of the concentration gradient is reduced by 40 and $85 \%$ for $D_{p}=10^{-7}$ and $D_{p}=10^{-8} \mathrm{~cm}^{2} \mathrm{~s}^{-1}$ at $300 \mathrm{~K}$, and by 30 and $82 \%$ for $D_{p}=10^{-8}$ and $D_{p}=10^{-9}$ $\mathrm{cm}^{2} \mathrm{~s}^{-1}$ at $200 \mathrm{~K}$. Slow diffusion in highly viscous liquid and semisolid states $\left(D_{p}<10^{-10} \mathrm{~cm}^{2} \mathrm{~s}^{-1}\right)$ cancels the effect of thermodynamic preferences, and result in uniform concentration profiles at both temperatures. Concentration profiles are not sensitive to varying RH, thus only one characteristic example is shown in Figure 6. Water's diffusion coefficient in h-CPA is estimated from separate unpublished MD simulations to be $10^{-5}-10^{-6} \mathrm{~cm}^{2} \mathrm{~s}^{-1}$ at $300 \mathrm{~K}$ depending on concentration, and $10^{-7}-10^{-8} \mathrm{~cm}^{2} \mathrm{~s}^{-1}$ at the lower temperature, similar values have been also reported based on experiments (Lienhard et al., 2015). This means that room temperature concentration profiles are virtually unaffected by bulk phase diffusion, as liquid-like diffusivities apply. Diffusion control is only probable for diffusion coefficients characteristic in semisolid particles. At $200 \mathrm{~K}$, the originally small concentration gradient is further reduced by slow diffusion. Bulk phase diffusion becomes the governing process for water uptake at low temperatures, regardless of the assumed value of the diffusion coefficient.

The quantitative description is only valid for the system studied and magnitudes may vary as a function of the composition of the organic shell. However, like main driving forces of the water uptake process, the formation of the concentration gradient can be expected in generic LLPS particles regardless of their actual chemical composition. The shape of the concentration profiles may change for a thicker organic layer having a bulk phase corresponding to a constant plateau in the free energy profile, which converts into a constant concentration region in the middle of the organic phase. Nevertheless, the maximum of the concentration profile coincides with the minimum of the free energy profile at the organic/water interface, which is determined predominantly by local entropy increase due to the lower density and increased conformational degrees of freedom, which is universal at boundaries between condensed phases. Similar considerations are valid for the minimum of the concentration profile, which is observed at the vapor/organic interface, whose value mainly depends on an interplay between intermolecular interactions, orientational order of the vapor/organic interface and interfacial entropy, which are largely insensitive to the thickness of the organic phase. The significant enrichment of the water/organic interfacial region in water may lead to a local dissolution of the organic phase. However, even when dissolution of the organic phase occurs, the strong preference of water molecules to be accommodated in the inner part of the organic shell results in depleted water concentrations at the surface, which ensures the presence of an organic rich film at the surface, and hence maintains low surface tensions despite of the elevated water concentration in the organic shell even when relative humidity approaches $100 \%$. This is consistent with recent experiments and model calculations which conclude that LLPS persists up to very high relative humidities (Liu et al., 2018). 


\subsubsection{Equilibrium saturation ratios}

The dynamics of water uptake affects the composition profile within the particle, hence the equilibrium vapor pressure of water over its evolution. For instance, kinetic hindrance of water molecules moving between organic shell and aqueous core will lead to higher water content in the outer shell than one in which such hindrances do not exist. Concentration profiles of water within the shell affect the water mole fraction and also droplet surface tension. Based on the findings from previous sections, three distinct scenarios through which LLPS may affect equilibrium saturation ratios of water are considered (Table 1). Given a fixed mass of dry substance, we use Köhler theory to calculate differences in equilibrium saturation ratios based on these assumptions how the driving force for water vapor condensation may be affected by chemical distribution and transport dynamics within an individual particle. Scenario (i) is valid at $300 \mathrm{~K}$ until the overall water concentration in the shell becomes too large for the

Table 1. Scenarios for Köhler theory calculations

\begin{tabular}{|c|c|c|c|c|}
\hline Scenario & Phases & Core/shell distr. of water & Shell composition & Surface tension \\
\hline (i) & LLPS & kinetically hindered & $\begin{array}{l}\text { nearly pure organic layer; } \\
\text { nonuniform water profile }\end{array}$ & pure CPA* \\
\hline (ii) & LLPS & kinetically hindered & mixed; uniform composition & saturated CPA solution ${ }^{* *}$ \\
\hline (iii) & LLPS & kinetic hindrance ignored & mixed; uniform composition & saturated CPA solution ${ }^{* *}$ \\
\hline (iv) & single & mixed; un & rm composition & pure water ${ }^{\dagger}$ \\
\hline \multicolumn{5}{|c|}{${ }^{*} \gamma=30 \mathrm{mN} \mathrm{m}^{-1}$ (Hyvärinen et al., 2006) } \\
\hline \multicolumn{5}{|c|}{${ }^{* *} \gamma=60 \mathrm{mN} \mathrm{m}^{-1}$ (Hyvärinen et al., 2006) } \\
\hline \multicolumn{5}{|c|}{${ }^{\dagger} \gamma=72 \mathrm{mN} \mathrm{m}^{-1}$ (Vargaftik et al., 1983) } \\
\hline
\end{tabular}

gradient to prevent the formation of a well mixed aqueous layer at the surface. Scenario (ii) is a model of the $200 \mathrm{~K}$ behavior as well as that of $300 \mathrm{~K}$ from the point where the concentration gradient cannot prevent the presence of a non-negligible amount of water in the surface layer. Scenario (iii) is a hypothetical case that provides a lower bound on the water content in the shell. Scenario (iv) provides a base case where the distribution of species are considered to be homogeneous throughout the particle. 


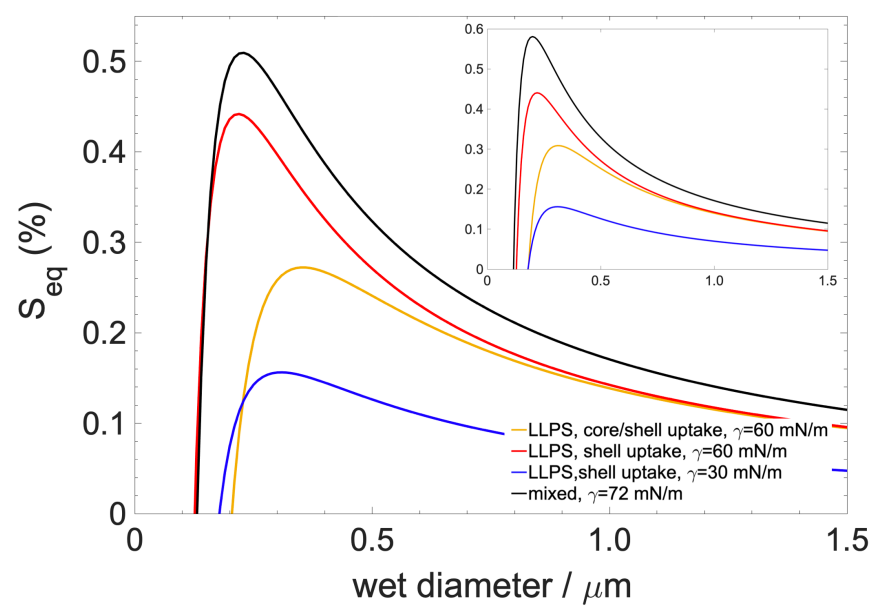

Figure 7. Köhler curves for $50 \mathrm{~nm}$ dry diameter particle containing $90 \%$ organic with $10 \%$ salt $\left(\left(\mathrm{NH}_{4}\right)_{2} \mathrm{SO}_{4}\right)$. The inset shows Köhler curves for a dry particle which contains organics only. Black curve: well mixed case; blue curve: scenario 1 (hindered core uptake with concentration gradient); red curve: scenario 2 (hindered core uptake without concentration gradient); yellow curve: scenario 3 (unhindered core uptake without concentration gradient)

Köhler curves are calculated for particles having a $50 \mathrm{~nm}$ dry diameter consisting of $90 \%$ organic compounds and $10 \%$ $\left(\left(\mathrm{NH}_{4}\right)_{2} \mathrm{SO}_{4}\right)$ and $100 \%$ organic with no salt (Figure 7). LLPS always tends to lower the critical supersaturation $\left(S_{c}\right)$ and increase the critical diameter $\left(d_{c}\right)$ compared to what is found for the well mixed case (iv). The lowering of the $S_{c} \sim 0.05$ $\%$ is the least pronounced if LLPS is preserved but only shell growth occurs, which is the scenario (iii) which describes the effect of hindered core uptake without concentration gradient. A moderate lowering of $S_{c} \sim 0.25 \%$ is found for the scenario in which LLPS maintains moderate surface tension (the surface is a saturated mixture of h-CPA and water) with uniform core and shell growth, that non-hindered core uptake (ii). Finally, $S_{c}$ is the lowest if the concentration gradient is taken into account (by assuming a pure organic surface) (i), in this case the critical supersaturation is reduced by $\sim 0.35 \%$. Trends are largely similar but more pronounced if the aqueous phase is assumed to contain only organics. In summary, LLPS — as a result of lower surface tensions characteristic of a saturated mixture - reduces critical supersaturations, while kinetic hindrance of the core uptake acts in the opposite direction since it increases the mole fraction of water in the organic shell. However, the concentration gradient and the resulting low surface tension characteristic of a pure or nearly pure organic surface imparts a strong effect, and invoke the strongest reduction in $S_{c}$ compared to the well mixed case among all the investigated scenarios.

\section{Conclusions}

Steered MD simulations of water uptake by a model LLPS aerosol particle consisting of a hydroxy-cis-pinonic acid surface layer and a pure aqueous core at two temperatures corresponding to the boundary layer ( $300 \mathrm{~K})$ and to the top of the troposphere ( $200 \mathrm{~K}$ ) were performed to investigate the mechanism of water uptake by LLPS aerosol via detailed analysis of the free energy 
profiles of the corresponding transfer process. In particular, the following questions were addressed: i) How does the uptake mechanism depend on temperature? ii) To what extent and under what conditions can water uptake by particles containing internal interfaces be described with a single uptake coefficient? iii) What role does the internal interface play in the water uptake mechanism? iv) How can the relationship between non-ideal mixing in LLPS particles and their increased CCN activity be explained on a molecular level?

These questions are answered using a novel combination of free energy profiles of interfacial transfer estimated from steered MD simulations and intrinsic surface analysis, which removes artificial smoothing and systematic errors caused by thermal fluctuations of liquid surfaces from estimates in interfacial properties and density profiles. Free energy profiles together with their entropic and energetic contributions were used to determine the water uptake mechanism, map the effect of the presence of an internal surface on the shape of the free energy, energy and entropy profiles and identify main thermodynamic driving forces behind the observed mechanism. Using steered MD in this context presents an advancement over previous approaches. The choice of the method can be rationalised by considering that each finite velocity pulling simulation used for estimating the free energy based on Jarzinsky's equality closely mimicks one realistic interfacial mass transfer event. The ensemble averaging ensures that a variety of potential pathways is included in the free energy estimate. Implications for realistic atmospheric processes were presented in the form of model calculations that link the molecular scale mechanism to the increased $\mathrm{CCN}$ activity of LLPS aerosol quantitatively for our model system and qualitatively for generic LLPS particles.

Our findings can be summarized as follows: i) The mechanism of water uptake (the shape of the free energy profile) is strongly temperature dependent. All minima and maxima can be attributed to entropic contributions, the minimum at the organic/water interface is due to a local maximum of the conformational entropy, while the subsequent maximum is a consequence of increased ordering of the water molecules in the first molecular layer of the aqueous phase. Due to the explicit temperature dependence of the weight of the entropy term in the free energy profile, features disappear at low temperature, however structural properties shaping entropy profiles are generally present in liquid/liquid and liquid/solid interfacial systems arbitrary composition. ii) Mass accommodation (vapor-to-organic transfer) coefficients were found to be near unity at both temperatures, which is in accordance with globally representative values. The core uptake (organic-to-water transfer) coefficient is reduced at room temperature $\left(k_{o / w}=0.05\right)$, while at low temperature $k_{o / w}=1$. This suggests that a single uptake coefficient is sufficient to describe the water uptake mechanism at $200 \mathrm{~K}$, while core uptake might have to be taken into account for the higher temperature. iii) Model estimates of shell and core uptake timescales revealed that depending on the particle phase diffusion coefficient shell uptake is at least four orders of magnitude faster that of core transfer. The slow diffusion of water in the organic shell can cause water to accumulate in the shell. This difference is further increased by 1-2 orders of magnitude if core uptake coefficient as a result of interfacial ordering of water molecules, leading to even more retention of water in the organic shell. iv) Converting free energy profiles into diffusion corrected concentration profiles allowed us to determine how molecular scale non-idealities in the solution structure can lead to enhanced surface activity. The molecular-scale explanation of the effect of non-ideal mixing on CCN activity lies in a non-uniform distribution of water molecules within the organic shell observed for liquid particles at $300 \mathrm{~K}$. The concentration distribution has a maximum near the organic/water interface, indicating that the condensing water molecules tend to accumulate near the aqueous phase and leaves the surface depleted in 
water. In other words, the observed concentration gradient maintains low surface tensions (nearly pure organic surface) and the LLPS state even when RH approaches $100 \%$. Köhler calculations reveal consequent reduced surface tensions are able to compensate the unfavorable effect on hindered core uptake on critical supersaturations.

In summary, our results point out that a single uptake coefficient is sufficient to describe water uptake in LLPS aerosol at low temperature, while at room temperature the models based on the complete uptake mechanism might be preferred. The effect of non-ideal mixing - usually accounted for in the form of Flory-Huggins parameter in continuum model calculations — are attributed to non-uniform distributions of the condensing water which maintain surface tension at low values even at high RH. The generalizability of thermodynamic driving forces suggests that the development of detailed models of aerosol growth kinetics incorporating these findings is possible, when combined with more rigorous and quantitative studies.

The strong temperature dependence of the water uptake mechanism, core uptake coefficient, as well as the presence nonuniform distribution of water within the organic shell at room temperature suggest that a detailed description of water uptake including these effects in a temperature dependent manner is necessary to improve aerosol growth kinetic models. The driving forces responsible for the typical features of the free energy profiles are generally valid for a wide range of liquid/vapor,liquid/liquid and liquid/solid surfaces. They presumably depend only weakly on the chemical nature of the organic compounds, which suggest that developing such a parametrization is feasible.

\section{Data availability}

PLUMED input files and starting configurations are available upon request. A movie showing a sample steered MD trajectory at $300 \mathrm{~K}$ is available at https://zenodo.org/record/4902870\#.YLsZwS0Rq_U.

\section{Appendix A: Structural analysis}

\section{A1 Intrinsic surface analysis and intrinsic density profiles}

We label molecules of the organic/water interface in a time resolved manner using the ITIM method (Sega et al., 2018). ITIM selects interfacial molecules by solely geometric criteria, thus it is considerably faster than alternative methods with essentially no loss in accuracy (Jorge et al., 2010). The ITIM method uses a probe sphere with a radius determined from the position of

565 the first peak of the corresponding radial distribution functions, in our case a value of $r=0.2 \mathrm{~nm}$ is used. The probe sphere is moved along a grid of testlines (a $200 \times 200$ grid is used in our analysis) perpendicular to the macroscopic plane of the interface. Once the probe sphere touches a an atom, the molecule to which it belongs to is labeled as interfacial. Contact is determined based a Pythagorean criterion. The list of surface molecules allows for selective estimation of various properties of 
the surface and the bulk and provides a means to reconstruct the intrinsic density profiles:

$570 \rho(z)=\frac{1}{A}\left\langle\sum_{i} \delta_{i}\left(z-z_{i}\right)+\xi\left(x_{i}, y_{i}\right)\right\rangle$

where $A$ is the macroscopic surface area of the interface, $x_{i}, y_{i}$ and $z_{i}$ are the Cartesian coordinates of the atoms constituting the system and $z$ is the position with respect to the local interface. $\xi\left(x_{i}, y_{i}\right) \sim k T / q^{2}$ is the capillary wave mode spectrum, with $q$ being the wave vector. In simple terms intrinsic density profiles are anchored to the first molecular layer of one of the condensed phase (the aqueous phase in our case), instead of being calculated along an external grid, thus they are able to resolve the near-surface fine structure of the density profiles which are otherwise washed away by capillary wave fluctuations. Intrinsic number density profile are used to compare free energy profiles with in a qualitative manner. The ITIM algorithm in particular allows for the separation of the surface molecules from those belonging to the bulk, and thus repeating the algorithm on the remaining bulk phase molecules can yield consecutive subsurface layers.

\section{Appendix B: Thermodynamic Analysis}

The free energy profiles were decomposed into energetic and various entropic contributions in order to understand the effects responsible for the features observed in the in the free energy profiles

\section{B1 Internal energy and hydrogen bonding}

The internal energy profile of the transfer process is estimated as the sum of the interaction energy between the pulled water molecule and the organic and water molecules weighted by the local mole fraction of the above two.

with $E_{i}(p, s)$ and $E_{i}(p, w)$ being the interaction energy between the pulled molecule and the solutes/water, calculated as the sum of short-term Coulombic and Lennard-Jones interactions (example values are listed in Table B1). $x_{s}^{l}(s)$ and $x_{w}^{l}(s)$ are local mole fraction profiles of the water and the organics. Local mole fraction profiles are calculated from the number of water and organic molecules found within $1 \mathrm{~nm}$ of the pulled molecule. The cutoff distance of $1 \mathrm{~nm}$ corresponds to the cutoff used in the simulations for short range interactions. This calculation is only plausible because solute/water interactions in the OPLS and TIP4P force fields were parametrized partially on quantum chemical calculations, thus individual interaction energies are physically meaningful. Equation B1 is evaluated for all realizations and averaged to yield the final profiles. Internal energy profiles of selected realizations are shown in Figure B1. 
https://doi.org/10.5194/acp-2021-488

Preprint. Discussion started: 17 June 2021

(c) Author(s) 2021. CC BY 4.0 License.

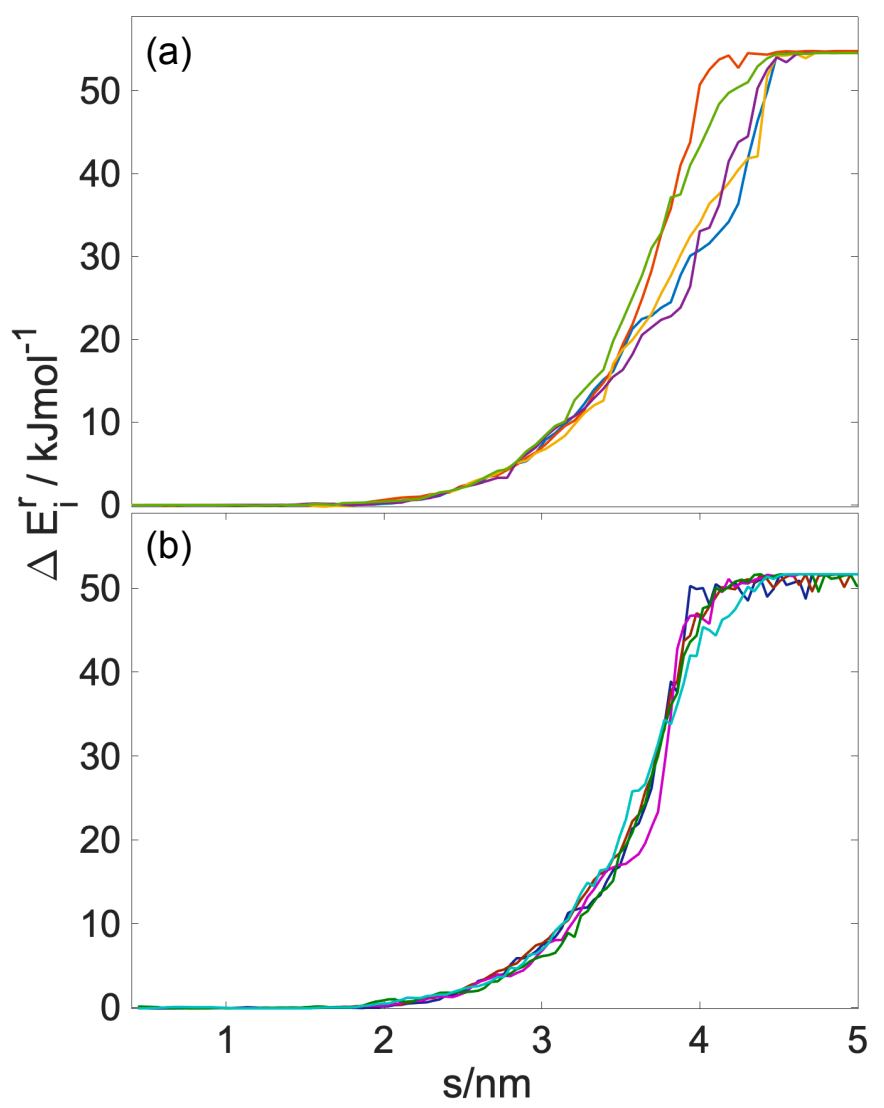

Figure B1. Internal energy profiles of selected realisations, a) $200 \mathrm{~K}$, b) $300 \mathrm{~K}$.

Table B1. Coulombic and Lennard-Jones contributions of the interaction energies in selected realisations

\begin{tabular}{|c|c|c|c|c|c|c|}
\hline & \multicolumn{2}{|c|}{$\mathrm{R} 1$} & \multicolumn{2}{|c|}{ R2 } & \multicolumn{2}{|c|}{ R3 } \\
\hline Type & $200 K$ & $300 K$ & $200 K$ & $300 K$ & $200 K$ & $300 K$ \\
\hline $\mathrm{LJ}(\mathrm{p}, \mathrm{s})$ & $0.2(0.1)$ & $-0.3(0.07)$ & $-0.43(0.2)$ & $-0.2(0.02)$ & $-0.09(0.02)$ & $-0.09(0.06)$ \\
\hline Coulomb(p,s) & $-5.7(1.3)$ & $-3.4(1.1)$ & $-5.4(2.1)$ & $-3.7(1.2)$ & $-6.8(3)$ & $-3.8(1.3)$ \\
\hline $\mathrm{LJ}(\mathrm{p}, \mathrm{w})$ & $14.4(1.9)$ & $11.3(1.5)$ & $15.1(2.2)$ & $11.1(1.4)$ & $14.2(2.1)$ & $10.9(1.4)$ \\
\hline Coulomb(p,w) & $-74.5(10.5)$ & $-66.5(8.5)$ & $-75.7(10.4)$ & $-66.4(8.2)$ & $-71.8(10.2)$ & $-65.6(1.0)$ \\
\hline
\end{tabular}




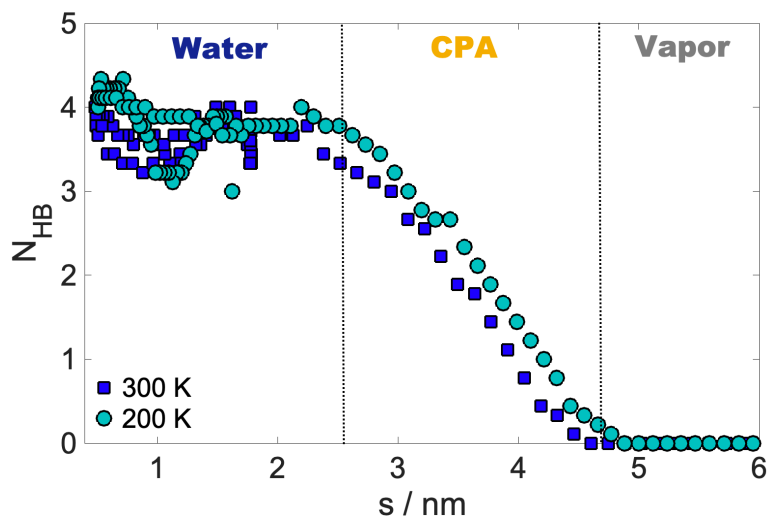

Figure B2. The number of hydrogen bonds formed by the pulled molecules along the reaction coordinate

The formation of hydrogen bonds is a major energetic driving force of the water uptake process. Figure B2 shows the number of total hydrogen bonds along the direction of the reaction coordinate.

\section{B2 Entropy}

\section{B2.1 Interfacial entropy}

Interfacial entropy accompanying any molecular transfer across phase boundaries can be calculated using the following formula 600 from statistical thermodynamics (Ward, 2002):

$$
\begin{aligned}
\frac{\Delta S_{I F}}{k}=4\left(1-\frac{T_{L}}{T_{V}}\right)+\left(\frac{1}{T_{L}}-\frac{1}{T_{V}}\right) \sum_{l=1}^{3}\left(\frac{\hbar \omega_{l}}{2 k}\right. & \left.+\frac{\hbar \omega_{l} / k}{\exp \left(\hbar \omega_{l} / k T_{V}\right)-1}\right)+ \\
& \quad+\frac{v_{L}^{s a t}}{k T_{L}}\left[P_{V}-P_{s a t}\left(T_{L}\right)\right]+\ln \left[\left(\frac{T_{L}}{T_{V}}\right)^{4}\left[\frac{P_{s a t}\left(T_{L}\right)}{P_{V}}\right]+\ln \left[\frac{q_{v i b}\left(T_{V}\right)}{q_{v i b}\left(T_{L}\right)}\right]\right],
\end{aligned}
$$

where $T_{V}$ and $T_{L}$ are the temperatures of the vapor and the liquid phase, $P_{s a t}$ and $P_{V}$ are the saturated and the actual vapor pressure, $v_{L}$ is the specific volume of the liquid phase $\omega_{i}$ are the vibrational frequencies and $q_{v i b}$ is the vibrational partition function. Figure B3 shows the modeled $T \Delta S_{I F}$ profiles at the two simulated temperatures, the values corresponding to the vapor pressures in the simulation box are highlighted with asterisks. The vapor pressure in the simulation box is estimated assuming the presence of the pulled molecule only in the vapor phase, using the universal gas law. 


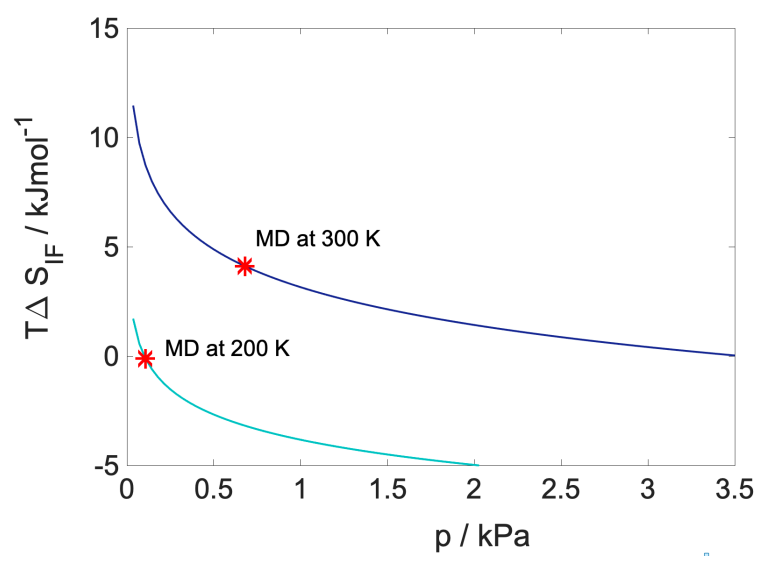

Figure B3. Modeled interfacial entropies at the simulated temperatures

\section{B2.2 Conformational entropy}

The conformational entropy profile is estimated as:

$610 S_{\text {conf }}=-k_{B} \sum_{i} x_{i}(s) \ln \left(x_{i}(s)\right)$ where $x_{i}(s)$ denotes mole fraction profiles of the components of the systems, with $s$ being the reaction coordinate used in the steered MD simulations.

\section{B2.3 Configurational entropy}

We calculate configurational entropy according to Schlitter's formula (Baron et al., 2006), thus based on the covariance matrix ((D)) of the atomic coordinates between two distinct groups of atoms, one being the pulled molecule and the other is either the ensemble of the solutes or waters constituting the bulk phase.

$S_{\text {config }}=\frac{k_{B}}{2} \ln \operatorname{det}\left(1+\frac{k_{B} T e^{2}}{\hbar} \mathbf{D}\right)$,

where $h$ is the Planck's constant divided by $2 \pi$ Two different contributions of the configurational entropy are considered i) between the pulled molecule and the solutes and between the pulled molecule and the solvents. In similar manner as for the internal energy, the weighted sum of these two yields the configurational entropy profile along the reaction coordinate $(s)$, with the weights being the local mole fractions of the water and the solute, whose calculation is described in the previous section.

\section{B2.4 Orientational entropy}

We propose an equation which can serve as a qualitative descriptor of the entropy related to the orientation of the molecules based on equations for translational (Bhandary et al., 2016) and translational-orientation entropy terms. (Piaggi and Parrinello, 
2018). With a simple exchange of the radial distribution function in (Bhandary et al., 2016) with the angular distribution function $(g(\theta))$ of the angle $(\theta)$ between the dipole vector of the water molecules and the surface normal axis, we obtain an expression for orientational entropy:

$S_{\text {or }}=-2 \pi k_{B} \int[g(\theta) \ln g(\theta)-g(\theta)+1] \sin \theta d \theta$

This expression is evaluated for angular distribution functions calculated in the first two molecular layers and the bulk of the aqueous phase, to highlight the effect of increased molecular order on the free energy profiles. The separation of interfacial water molecules and those constituting the second layer is performed by two consecutive repetitions of the ITIM algorithm, using the output bulk phase of the first one as input for the second one.

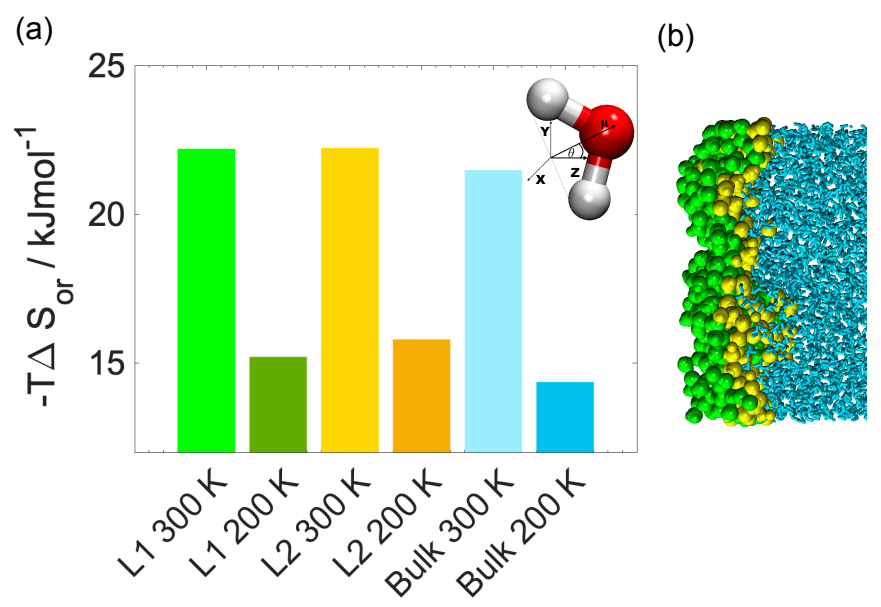

Figure B4. a) Orientational entropies in the first and second molecular layers and the bulk phase for both simulated temperatures. Inset: the definition of the $\theta$ angle. b) Snapshot from a $300 \mathrm{~K}$ realisation showing the first two molecular layers of the aqueous phase in green (L1) and yellow (L2).

At both temperatures the bulk phase orientational entropy is higher ( $-T \Delta S$ is lower) than in the first two layers due to stronger ordering in the first two interfacial layers (Figure B4). We note that equation B5 cannot completely describe the entropy loss due to preferential ordering of the water molecules at the interface since due to its point group symmetry $\left(C_{2 v}\right)$, the orientation of water molecules with respect to an external vector or plane cannot be described with a single angle, instead the joint distribution of two angles is necessary. The development of an adapted expression of the orientational entropy of such cases is however out of the scope of this study. The one dimensional representation is incomplete and thus gives only a qualitative insight but the temperature dependence of $T \Delta S_{\text {or }}$ within the layers is clear, the extension of the orientational entropy formula to multiple dimensions is part of ongoing work. To complete the description of orientational differences between the surface and the bulk of the aqueous phase, we calculate joint distributions of angles $\cos \theta$ ' and $\phi$, which are chosen to fully describe the orientation of water molecules with respect to the normal vector of the macroscopic surface(Bartók-Pártay et al., 2008), in the first two molecular layers and the bulk. $\theta^{\prime}$ and $\phi$ are defined in a Cartesian frame centered on the water 
molecules, the $\mathbf{z}$ axis points from the water oxygen towards the midpoint of the segment connecting the hydrogen atoms, the $\mathbf{y}$ axis is parallel to that segment, and the $\mathbf{x}$ axis is perpendicular to both $\mathbf{z}$ and $\mathbf{y} . \theta^{\prime}$ is the angle between the macroscopic surface normal vector $(\mathbf{Z})$ and the molecule centered $\mathbf{z}$ axis, and $\phi$ is the angle between the $\mathbf{x}$ axis and the projection of the surface normal vector to the $\mathbf{x}, \mathbf{y}$ plane.

(a)

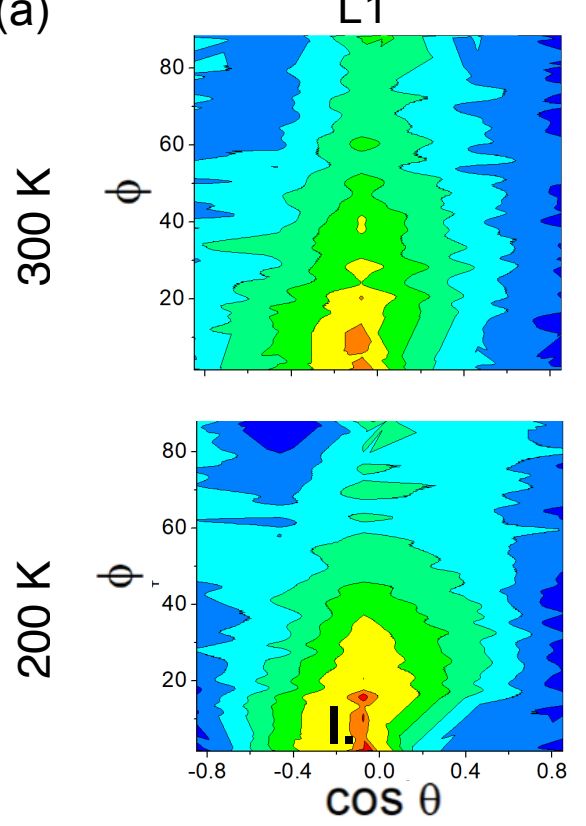

L2
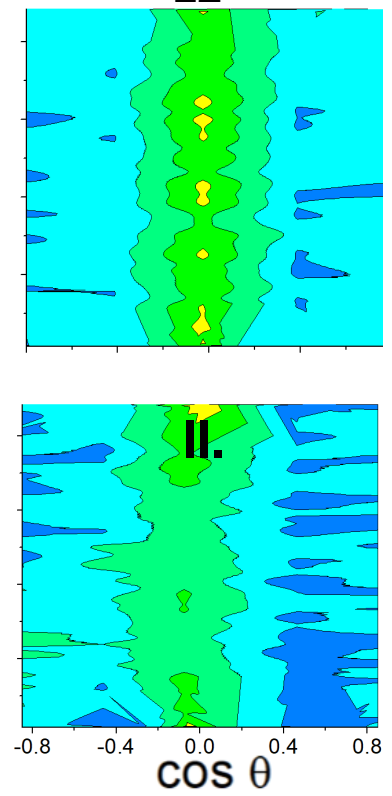

(c)

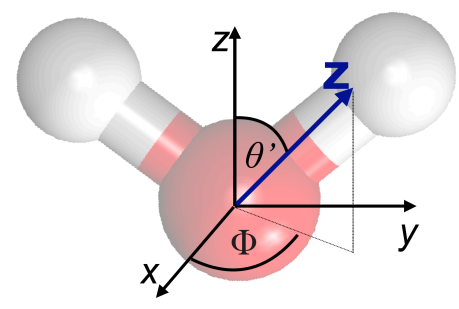

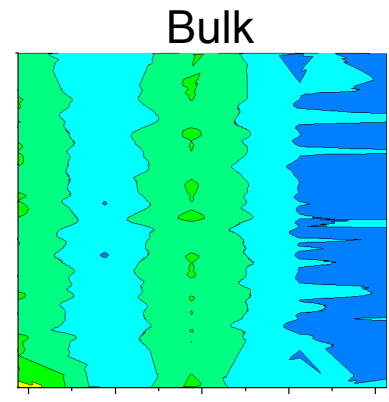
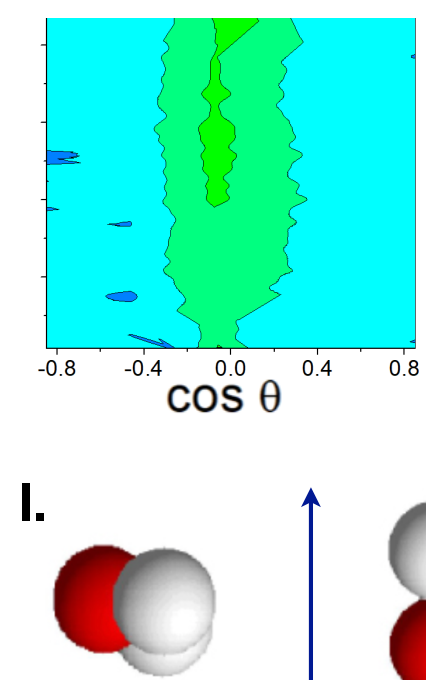
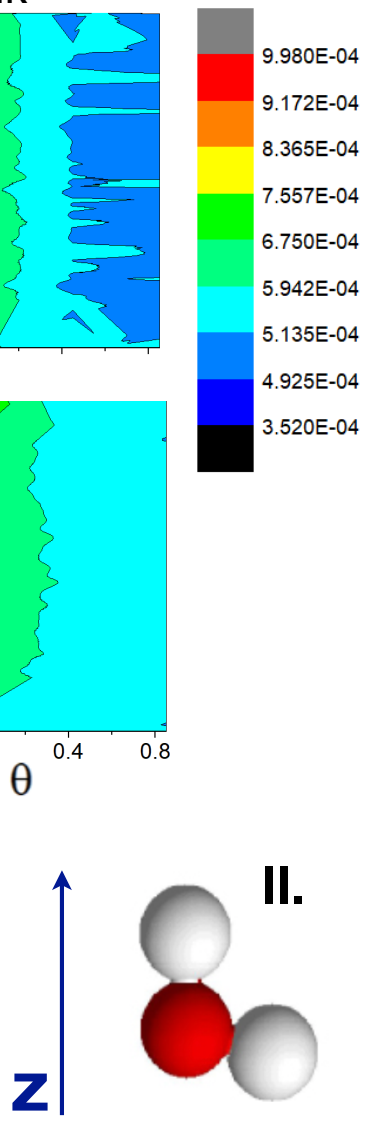

Figure B5. (a) Orientational maps of the water molecules in the first (L1), second (L2) molecular layer and the bulk phase. (b) The definition of $\theta^{\prime}$ and $\phi$ in the Cartesian frame centered on a water. (c) Examples of the two main orientations.

Figure B5 shows orientational maps in the first two molecular layers and the bulk of the aqueous phase. In the first layer water molecules show a very strong preference to be aligned with dipole vectors parallel to the surface or slightly tilted towards the bulk aqueous phase, orientation I. in Figure B5 a) and c). In another distinguished orientation, which appears in the first and second layer at $300 \mathrm{~K}$ and the second layer at $200 \mathrm{~K}$, one $\mathrm{O}-\mathrm{H}$ bond points in the direction of the surface normal vector and the other slightly inwards to the bulk aqueous phase. Orientational preferences diminish progressively when moving towards the bulk phase, indicating the decrease of orientational order and thus a increase in orientational entropy. It is 
https://doi.org/10.5194/acp-2021-488

Preprint. Discussion started: 17 June 2021

(c) Author(s) 2021. CC BY 4.0 License.

(c) (1)

a remarkable difference between our system and aqueous interfaces of hydrophobic organic compounds (dichloromethane and dichloroethane) studied previously (Hantal et al., 2010), where preferred orientations were only found in the first molecular layer of water in direct contact with the organic phase, that the second layer is more ordered than the bulk phase, it is due to the fact that h-CPA mixes more readily with water then hydrophobic organics, thus h-CPA molecules can penetrate into the second and third molecular layer as well into the bulk phase, and contact with the dissolved organic molecules promotes orientations that are similar to those found at the interface. We also note that the preferred orientations found of interfacial waters are universal across a large spectra of organic/water interfaces.

Author contributions. M.D designed and performed simulations and analysis and wrote the manuscript. S.T. and A.N. contributed to analysis, interpreted results and wrote the manuscript.

Competing interests. The authors declare no competing interests 


\section{References}

Abraham, M. J., Murtola, T., Schulz, R., Páll, S., Smith, J. C., Hess, B., and Lindahl, E.: GROMACS: High Performance Molecular Simulations through Multi-level Parallelism from Laptops to Supercomputers, SoftwareX, 1-2, 19 - 25, 2015.

Allen, W. J., Wiley, M. R., Myles, K. M., Adelman, Z. N., and Bevan, D. R.: Steered molecular dynamics identifies critical residues of the Nodamura virus B2 suppressor of RNAi, Journal of Molecular Modeling, 20, 2092, https://doi.org/10.1007/s00894-014-2092-0, https: //doi.org/10.1007/s00894-014-2092-0, 2014.

Bahadur, R. and Russell, L.: Water uptake coefficients and deliquescence of $\mathrm{NaCl}$ nanoparticles at atmospheric relative humidities from molecular dynamics simulations, The Journal of chemical physics, 129, 094 508, https://doi.org/10.1063/1.2971040, 2008.

Baron, R., de Vries, A. H., Hünenberger, P. H., and van Gunsteren, W. F.: Configurational Entropies of Lipids in Pure and Mixed Bilayers from Atomic-Level and Coarse-Grained Molecular Dynamics Simulations, The Journal of Physical Chemistry B, 110, 15 602-15 614, https://doi.org/10.1021/jp061627s, https://doi.org/10.1021/jp061627s, pMID: 16884285, 2006.

Bartók-Pártay, L., Horvai, G., and Jedlovszky, P.: Molecular level structure of the liquid/liquid interface. Molecular dynamics simulation and ITIM analysis of the water-CCl4 system, Physical chemistry chemical physics : PCCP, 10, 4754-64, https://doi.org/10.1039/b807299j, 2008.

Benjamin, I.: Hydronium ion at the water/1, 2-dichloroethane interface: Structure, thermodynamics, and dynamics of ion transfer, The Journal of chemical physics, 151, $094701,2019$.

Benjamin, 1.: Mechanism and dynamics of ion transfer across a liquid-liquid interface, Science, 261, 1558-1560, 1993.

Bhandary, D., Benková, Z., Cordeiro, M. N. D. S., and Singh, J. K.: Molecular dynamics study of wetting behavior of grafted thermo-responsive PNIPAAm brushes, Soft Matter, 12, 3093-3102, https://doi.org/10.1039/C5SM02684A, http://dx.doi.org/10.1039/ C5SM02684A, 2016.

Braga, C., Muscatello, J., Lau, G., Müller, E. A., and Jackson, G.: Nonequilibrium study of the intrinsic free-energy profile across a liquidvapour interface, The Journal of Chemical Physics, 144, 044 703, https://doi.org/10.1063/1.4940137, https://doi.org/10.1063/1.4940137, 2016.

Bussi, G., Donadio, D., and Parrinello, M.: Canonical Sampling through Velocity Rescaling, J. Chem. Phys., $126,014101,2007$.

Bzdek, B. R. and Reid, J. P.: Perspective: Aerosol microphysics: From molecules to the chemical physics of aerosols, The Journal of Chemical Physics, 147, 220 901, https://doi.org/10.1063/1.5002641, https://doi.org/10.1063/1.5002641, 2017.

Cipcigan, F., Sokhan, V., Jones, A., Crain, J., and Martyna, G.: Hydrogen bonding and molecular orientation at the liquid-vapour interface of water, Phys. Chem. Chem. Phys., 17, https://doi.org/10.1039/C4CP05506C, 2015.

Clement, C. F., Kulmala, M., and Vesala, T.: Theoretical consideration on sticking probabilities, Journal of Aerosol Science, 27, 869 882, https://doi.org/https://doi.org/10.1016/0021-8502(96)00032-8, http://www.sciencedirect.com/science/article/pii/0021850296000328, fuchs Memorial Issue, 1996.

Cuendet, M. A.: The Jarzynski identity derived from general Hamiltonian or non-Hamiltonian dynamics reproducing NVT or NPT ensembles, The Journal of chemical physics, 125, 144 109, 2006.

Darvas, M., Pojják, K., Horvai, G., and Jedlovszky, P.: Molecular dynamics simulation and identification of the truly interfacial molecules (ITIM) analysis of the liquid-vapor interface of dimethyl sulfoxide, The Journal of Chemical Physics, 132, 134701, https://doi.org/10.1063/1.3368111, https://doi.org/10.1063/1.3368111, 2010a. 
Darvas, M., Pártay, L. B., Jedlovszky, P., and Horvai, G.: Computer simulation and ITIM analysis of the surface of water-methanol mixtures containing traces of water, Journal of Molecular Liquids, 153, 88 - 93, https://doi.org/https://doi.org/10.1016/j.molliq.2009.06.004, http: //www.sciencedirect.com/science/article/pii/S0167732209001287, 2010b.

Darvas, M., Gilányi, T., and Jedlovszky, P.: Competitive Adsorption of Surfactants and Polymers at the Free Water Surface. A Computer Simulation Study of the Sodium Dodecyl Sulfate-Poly(ethylene oxide) System, The Journal of Physical Chemistry B, 115, 933-944, https://doi.org/10.1021/jp110270c, https://doi.org/10.1021/jp110270c, pMID: 21250730, 2011a.

Darvas, M., Jorge, M., D. S. Cordeiro, M. N., and Jedlovszky, P.: Solvation Free Energy Profile of the SCN- Ion across the Water-1,2Dichloroethane Liquid/Liquid Interface. A Computer Simulation Study, The Journal of Physical Chemistry C, 115, 11 140-11 146, https://doi.org/10.1021/jp2018605, https://doi.org/10.1021/jp2018605, 2011 b.

Darvas, M., Jorge, M., Cordeiro, M. N. D. S., Kantorovich, S. S., Sega, M., and Jedlovszky, P.: Calculation of the Intrinsic Solvation Free Energy Profile of an Ionic Penetrant Across a Liquid-Liquid Interface with Computer Simulations, The Journal of Physical Chemistry B, 117, 16 148-16 156, https://doi.org/10.1021/jp404699t, https://doi.org/10.1021/jp404699t, pMID: 24175995, 2013.

Davidovits, P., Worsnop, D., Jayne, J., Kolb, C., Winkler, P., Vrtala, A., Wagner, P., Kulmala, M., Lehtinen, K., Vesala, T., et al.: Mass accommodation coefficient of water vapor on liquid water, 2004.

Davies, J., Miles, R., Haddrell, A., and Reid, J.: Influence of organic films on the evaporation and condensation of water in aerosol, Proceedings of the National Academy of Sciences of the United States of America, https://doi.org/10.1073/pnas.1305277110, 2013.

Diveky, M. E., Roy, S., Cremer, J. W., David, G., and Signorell, R.: Assessing relative humidity dependent photoacoustics to retrieve mass accommodation coefficients of single optically trapped aerosol particles, Phys. Chem. Chem. Phys., 21, 4721-4731, https://doi.org/10.1039/C8CP06980H, http://dx.doi.org/10.1039/C8CP06980H, 2019.

Ergin, G. and Takahama, S.: Carbon Density Is an Indicator of Mass Accommodation Coefficient of Water on Organic-Coated Water Surface, The Journal of Physical Chemistry A, 120, 2885-2893, https://doi.org/10.1021/acs.jpca.6b01748, https://doi.org/10.1021/acs.jpca. 6b01748, pMID: 27089481, 2016.

Essmann, U., Perera, L., Berkowitz, M. L., Darden, T., Lee, H., and Pedersen, L. G.: A Smooth Particle Mesh Ewald Method, J. Chem. Phys., 103, 8577-8593, 1995.

Facchini, M. C., Mircea, M., Fuzzi, S., and Charlson, R. J.: Cloud albedo enhancement by surface-active organic solutes in growing droplets, Nature, 401, 257-259, https://doi.org/10.1038/45758, https://doi.org/10.1038/45758, 1999.

Fuzzi, S., Andreae, M. O., Huebert, B. J., Kulmala, M., Bond, T. C., Boy, M., Doherty, S. J., Guenther, A., Kanakidou, M., Kawamura, K., Kerminen, V.-M., Lohmann, U., Russell, L. M., and Pöschl, U.: Critical assessment of the current state of scientific knowledge, terminology, and research needs concerning the role of organic aerosols in the atmosphere, climate, and global change, Atmospheric Chemistry and Physics, 6, 2017-2038, https://doi.org/10.5194/acp-6-2017-2006, https://www.atmos-chem-phys.net/6/2017/2006/, 2006.

Ghatee, M. H., Zolghadr, A. R., Moosavi, F., and Pakdel, L.: The extent of molecular orientation at liquid/vapor interface of pyridine and its alkyl derivatives by molecular dynamics simulation, The Journal of Chemical Physics, 134, 074 707, https://doi.org/10.1063/1.3554361, https://doi.org/10.1063/1.3554361, 2011.

Gore, J., Ritort, F., and Bustamante, C.: Bias and error in estimates of equilibrium free-energy differences from nonequilibrium measurements, Proceedings of the National Academy of Sciences, 100, 12 564-12 569, https://doi.org/10.1073/pnas.1635159100, https: //www.pnas.org/content/100/22/12564, 2003.

740 Gorkowski, K., Donahue, N. M., and Sullivan, R. C.: Emulsified and liquid-liquid phase-separated states of $\alpha$-pinene secondary organic aerosol determined using aerosol optical tweezers, Environmental Science \& Technology, 51, 12 154-12 163, 2017. 
Gorkowski, K., Donahue, N. M., and Sullivan, R. C.: Aerosol Optical Tweezers Constrain the Morphology Evolution of Liquid-Liquid Phase-Separated Atmospheric Particles, Chem, 6, 204-220, https://doi.org/https://doi.org/10.1016/j.chempr.2019.10.018, https://www. sciencedirect.com/science/article/pii/S2451929419304723, 2020.

Grote, R. F. and Hynes, J. T.: The stable states picture of chemical reactions. II. Rate constants for condensed and gas phase reaction models, The Journal of Chemical Physics, 73, 2715-2732, 1980.

Hantal, G., Darvas, M., Pártay, L. B., Horvai, G., and Jedlovszky, P.: Molecular level properties of the free water surface and different organic liquid/water interfaces, as seen from ITIM analysis of computer simulation results, Journal of Physics: Condensed Matter, 22, 284112 , https://doi.org/10.1088/0953-8984/22/28/284112, https://doi.org/10.1088\%2F0953-8984\%2F22\%2F28\%2F284112, 2010.

Hummer, G. and Szabo, A.: Free energy reconstruction from nonequilibrium single-molecule pulling experiments, Proceedings of the National Academy of Sciences, 98, 3658-3661, https://doi.org/10.1073/pnas.071034098, https://www.pnas.org/content/98/7/3658, 2001.

Hyvärinen, A.-P., Lihavainen, H., Gaman, A., Vairila, L., Ojala, H., Kulmala, M., and Viisanen, Y.: Surface Tensions and Densities of Oxalic, Malonic, Succinic, Maleic, Malic, and cis-Pinonic Acids, Journal of Chemical \& Engineering Data, 51, 255-260, https://doi.org/10.1021/je050366x, https://doi.org/10.1021/je050366x, 2006.

IPCC, .: Summary for policymakers.

Jarzynski, C.: Nonequilibrium Equality for Free Energy Differences, , 78, 2690-2693, https://doi.org/10.1103/PhysRevLett.78.2690, 1997.

Johansson, S. M., Lovrić, J., Kong, X., Thomson, E. S., Hallquist, M., and Pettersson, J. B. C.: Experimental and Computational Study of Molecular Water Interactions with Condensed Nopinone Surfaces Under Atmospherically Relevant Conditions, The Journal of Physical Chemistry A, 0, null, https://doi.org/10.1021/acs.jpca.9b10970, https://doi.org/10.1021/acs.jpca.9b10970, pMID: 32281376, 2020.

Jorge, M., Hantal, G., Jedlovszky, P., and Cordeiro, M. N. D. S.: A Critical Assessment of Methods for the Intrinsic Analysis of Liquid Interfaces: 2. Density Profiles, The Journal of Physical Chemistry C, 114, 18 656-18 663, https://doi.org/10.1021/jp107378s, https://doi. org/10.1021/jp107378s, 2010.

Jorgensen, W. L. and Tirado-Rives, J.: The OPLS [Optimized Potentials for Liquid Simulations] Potential Functions for Proteins, Energy Minimizations for Crystals of Cyclic Peptides and Crambin, J. Am. Chem. Soc., 110, 1657-1666, 1988.

Jorgensen, W. L., Chandrasekhar, J., Madura, J. D., Impey, R. W., and Klein, M. L.: Comparison of Simple Potential Functions for Simulating Liquid Water, J. Chem. Phys., 79, 926-935, 1983.

Julin, J., Winkler, P., Donahue, N., Wagner, P., and Riipinen, I.: Near-Unity Mass Accommodation Coefficient of Organic Molecules of Varying Structure, Environmental science technology, 48, https://doi.org/10.1021/es501816h, 2014.

Karnes, J. J. and Benjamin, I.: Geometric and energetic considerations of surface fluctuations during ion transfer across the water-immiscible organic liquid interface, The Journal of chemical physics, 145, 014 701, 2016.

Kertész, J., Darvas, M., Jedlovszky, P., and Horvai, G.: Reprint of "Role of the fluidity of a liquid phase in determining the surface properties of the opposite phase", Journal of Molecular Liquids, 189, 122 - 128, https://doi.org/https://doi.org/10.1016/j.molliq.2013.06.009, http: //www.sciencedirect.com/science/article/pii/S0167732213002110, fluid phase associations, 2014.

Klug, J., Triguero, C., Del Pópolo, M. G., and Tribello, G. A.: Using Intrinsic Surfaces To Calculate the Free-Energy Change When Nanoparticles Adsorb on Membranes, The Journal of Physical Chemistry B, 122, 6417-6422, 2018.

Krechmer, J. E., Day, D. A., Ziemann, P. J., and Jimenez, J. L.: Direct measurements of gas/particle partitioning and mass accommodation coefficients in environmental chambers, Environmental science \& technology, 51, 11 867-11 875, 2017.

Krieger, U. K., Marcolli, C., and Reid, J. P.: Exploring the complexity of aerosol particle properties and processes using single particle techniques, Chem. Soc. Rev., 41, 6631-6662, https://doi.org/10.1039/C2CS35082C, http://dx.doi.org/10.1039/C2CS35082C, 2012. 

a Function of Temperature, The Journal of Physical Chemistry A, 105, 10627-10 634, https://doi.org/10.1021/jp012758q, https://doi.org/ $10.1021 / j p 012758 \mathrm{q}, 2001$.

Lienhard, D. M., Huisman, A. J., Krieger, U. K., Rudich, Y., Marcolli, C., Luo, B. P., Bones, D. L., Reid, J. P., Lambe, A. T., Canagaratna, M. R., Davidovits, P., Onasch, T. B., Worsnop, D. R., Steimer, S. S., Koop, T., and Peter, T.: Viscous organic aerosol particles in the upper troposphere: diffusivity-controlled water uptake and ice nucleation?, Atmospheric Chemistry and Physics, 15, 13 599-13613, https://doi.org/10.5194/acp-15-13599-2015, https://www.atmos-chem-phys.net/15/13599/2015/, 2015.

Liu, P., Song, M., Zhao, T., Gunthe, S. S., Ham, S., He, Y., Qin, Y. M., Gong, Z., Amorim, J. C., Bertram, A. K., and Martin, S. T.: Resolving the mechanisms of hygroscopic growth and cloud condensation nuclei activity for organic particulate matter, Nature Communications, 9 , 4076, https://doi.org/10.1038/s41467-018-06622-2, https://doi.org/10.1038/s41467-018-06622-2, 2018.

Liu, X., Day, D. A., Krechmer, J. E., Brown, W., Peng, Z., Ziemann, P. J., and Jimenez, J. L.: Direct measurements of semi-volatile organic compound dynamics show near-unity mass accommodation coefficients for diverse aerosols, Communications Chemistry, 2, 98, https://doi.org/10.1038/s42004-019-0200-x, https://doi.org/10.1038/s42004-019-0200-x, 2019.

Makowski, M. J., Stern, A. C., Hemminger, J. C., and Tobias, D. J.: Orientation and Structure of Acetonitrile in Water at the Liquid-Vapor Interface: A Molecular Dynamics Simulation Study, The Journal of Physical Chemistry C, 120, 17555-17563, https://doi.org/10.1021/acs.jpcc.6b05448, https://doi.org/10.1021/acs.jpcc.6b05448, 2016.

Miles, R. E. H., Davies, J. F., and Reid, J. P.: The influence of the surface composition of mixed monolayer films on the evaporation coefficient of water, Phys. Chem. Chem. Phys., 18, 19847-19 858, https://doi.org/10.1039/C6CP03826C, http://dx.doi.org/10.1039/C6CP03826C, 2016.

Moore, R. H., Raatikainen, T., Langridge, J. M., Bahreini, R., Brock, C. A., Holloway, J. S., Lack, D. A., Middlebrook, A. M., Perring, A. E., Schwarz, J. P., et al.: CCN spectra, hygroscopicity, and droplet activation kinetics of secondary organic aerosol resulting from the 2010 Deepwater Horizon oil spill, Environmental science \& technology, 46, 3093-3100, 2012.

Morales Betancourt, R. and Nenes, A.: Understanding the contributions of aerosol properties and parameterization discrepancies to droplet number variability in a global climate model, Atmospheric Chemistry and Physics, 14, 4809-4826, https://doi.org/10.5194/acp-14-48092014, https://www.atmos-chem-phys.net/14/4809/2014/, 2014.

Morita, A., Sugiyama, M., Kameda, H., Koda, S., and Hanson, D. R.: Mass Accommodation Coefficient of Water: Molecular Dynamics Simulation and Revised Analysis of Droplet Train/Flow Reactor Experiment, The Journal of Physical Chemistry B, 108, 9111-9120, https://doi.org/10.1021/jp030479s, 2004.

Noziere, B.: Don't forget the surface, Science, 351, 1396-1397, https://doi.org/10.1126/science.aaf3253, https://science.sciencemag.org/ content/351/6280/1396, 2016.

810 Ovadnevaite, J., Zuend, A., Laaksonen, A., Sanchez, K. J., Roberts, G., Ceburnis, D., Decesari, S., Rinaldi, M., Hodas, N., Facchini, M. C., Seinfeld, J. H., and O'Dowd, C.: Surface tension prevails over solute effect in organic-influenced cloud droplet activation, Nature, 546, 637-641, https://doi.org/10.1038/nature22806, https://doi.org/10.1038/nature22806, 2017.

Pajunoja, A., Lambe, A. T., Hakala, J., Rastak, N., Cummings, M. J., Brogan, J. F., Hao, L., Paramonov, M., Hong, J., Prisle, N. L., Malila, J., Romakkaniemi, S., Lehtinen, K. E. J., Laaksonen, A., Kulmala, M., Massoli, P., Onasch, T. B., Donahue, N. M., Riipinen, I., Davidovits, P., Worsnop, D. R., Petäjä, T., and Virtanen, A.: Adsorptive uptake of water by semisolid secondary organic aerosols, Geophysical Research Letters, 42, 3063-3068, https://doi.org/10.1002/2015GL063142, https://agupubs.onlinelibrary.wiley.com/doi/abs/10.1002/ 2015GL063142, 2015. 
Park, S. and Schulten, K.: Calculating potentials of mean force from steered molecular dynamics simulation, The Journal of chemical physics, 120, 5946-61, https://doi.org/10.1063/1.1651473, 2004.

Pártay, L. B., Jedlovszky, P., Vincze, A., and Horvai, G.: Properties of Free Surface of Water-Methanol Mixtures. Analysis of the Truly Interfacial Molecular Layer in Computer Simulation, The Journal of Physical Chemistry B, 112, 5428-5438, https://doi.org/10.1021/jp711547e, https://doi.org/10.1021/jp711547e, pMID: 18393551, 2008.

Petters, M. D., Kreidenweis, S. M., Snider, J. R., Koehler, K. A., Wang, Q., Prenni, A. J., and Demott, P. J.: Cloud droplet activation of polymerized organic aerosol, Tellus B: Chemical and Physical Meteorology, 58, 196-205, https://doi.org/10.1111/j.1600-0889.2006.00181.x, https://doi.org/10.1111/j.1600-0889.2006.00181.x, 2006.

Piaggi, P. M. and Parrinello, M.: Predicting polymorphism in molecular crystals using orientational entropy, Proceedings of the National Academy of Sciences, 115, 10 251-10 256, https://doi.org/10.1073/pnas.1811056115, https://www.pnas.org/content/115/41/10251, 2018.

Pojják, K., Darvas, M., Horvai, G., and Jedlovszky, P.: Properties of the Liquid-Vapor Interface of Water-Dimethyl Sulfoxide Mixtures. A Molecular Dynamics Simulation and ITIM Analysis Study, The Journal of Physical Chemistry C, 114, 12207-12220, https://doi.org/10.1021/jp101442m, https://doi.org/10.1021/jp101442m, 2010.

Prenni, A. J., Petters, M. D., Kreidenweis, S. M., DeMott, P. J., and Ziemann, P. J.: Cloud droplet activation of secondary organic aerosol, Journal of Geophysical Research: Atmospheres, 112, https://doi.org/10.1029/2006JD007963, https://agupubs.onlinelibrary.wiley.com/doi/ abs/10.1029/2006JD007963, 2007.

Pruppacher, H. and Klett, J.: Microstructure of Atmospheric Clouds and Precipitation, pp. 10-73, Springer Netherlands, Dordrecht, https://doi.org/10.1007/978-0-306-48100-0_2, https://doi.org/10.1007/978-0-306-48100-0_2, 2010.

Raatikainen, T., Nenes, A., Seinfeld, J. H., Morales, R., Moore, R. H., Lathem, T. L., Lance, S., Padró, L. T., Lin, J. J., Cerully, K. M., Bougiatioti, A., Cozic, J., Ruehl, C. R., Chuang, P. Y., Anderson, B. E., Flagan, R. C., Jonsson, H., Mihalopoulos, N., and Smith, J. N.: Worldwide data sets constrain the water vapor uptake coefficient in cloud formation, Proceedings of the National Academy of Sciences, 110, 3760-3764, https://doi.org/10.1073/pnas.1219591110, https://www.pnas.org/content/110/10/3760, 2013.

Renbaum-Wolff, L., Song, M., Marcolli, C., Zhang, Y., Liu, P. F., Grayson, J. W., Geiger, F. M., Martin, S. T., and Bertram, A. K.: Observations and implications of liquid-liquid phase separation at high relative humidities in secondary organic material produced by $\alpha$-pinene ozonolysis without inorganic salts, Atmospheric Chemistry and Physics, 16, 7969-7979, https://doi.org/10.5194/acp-16-79692016, https://www.atmos-chem-phys.net/16/7969/2016/, 2016.

Rowlinson, J. S. J. S.: Molecular theory of capillarity / by J.S. Rowlinson and B. Widom., International series of monographs on chemistry ; 8, Clarendon Press, 1982.

Roy, S., Diveky, M. E., and Signorell, R.: Mass Accommodation Coefficients of Water on Organics from Complementary Photoacoustic and Light Scattering Measurements on Laser-Trapped Droplets, The Journal of Physical Chemistry C, 124, 2481-2489, https://doi.org/10.1021/acs.jpcc.9b09934, https://doi.org/10.1021/acs.jpcc.9b09934, 2020.

Ruehl, C. R., Chuang, P. Y., Nenes, A., Cappa, C. D., Kolesar, K. R., and Goldstein, A. H.: Strong evidence of surface tension reduction in microscopic aqueous droplets, Geophysical Research Letters, 39, https://doi.org/10.1029/2012GL053706, https://agupubs.onlinelibrary. wiley.com/doi/abs/10.1029/2012GL053706, 2012.

Ruehl, C. R., Davies, J. F., and Wilson, K. R.: An interfacial mechanism for cloud droplet formation on organic aerosols, Science, 351, 1447-1450, https://doi.org/10.1126/science.aad4889, https://science.sciencemag.org/content/351/6280/1447, 2016.

Sakaguchi, S. and Morita, A.: Mass accommodation mechanism of water through monolayer films at water/vapor interface, The Journal of Chemical Physics, 137, 064 701, https://doi.org/10.1063/1.4740240, https://doi.org/10.1063/1.4740240, 2012. 
Saleh, R., Donahue, N. M., and Robinson, A. L.: Time Scales for Gas-Particle Partitioning Equilibration of Secondary Organic Aerosol Formed from Alpha-Pinene Ozonolysis, Environmental Science \& Technology, 47, 5588-5594, https://doi.org/10.1021/es400078d, https: //doi.org/10.1021/es400078d, pMID: 23647198, 2013.

Sareen, N., Schwier, A. N., Lathem, T. L., Nenes, A., and McNeill, V. F.: Surfactants from the gas phase may promote cloud droplet formation, Proceedings of the National Academy of Sciences, 110, 2723-2728, https://doi.org/10.1073/pnas.1204838110, https://www. pnas.org/content/110/8/2723, 2013.

Schöll-Paschinger, E. and Dellago, C.: A proof of Jarzynski's nonequilibrium work theorem for dynamical systems that conserve the canonical distribution, The Journal of chemical physics, 125, 054 105, 2006.

Sega, M., Hantal, G., Fábián, B., and Jedlovszky, P.: Pytim: A python package for the interfacial analysis of molecular simulations, Journal of Computational Chemistry, 39, https://doi.org/10.1002/jcc.25384, 2018.

Shiraiwa, M. and Pöschl, U.: Mass Accommodation and Gas-Particle Partitioning in Secondary Organic Aerosols: Dependence on Diffusivity, Volatility, Particle-phase Reactions, and Penetration Depth, Atmospheric Chemistry and Physics Discussions, 2020, 1-30, https://doi.org/10.5194/acp-2020-536, https://www.atmos-chem-phys-discuss.net/acp-2020-536/, 2020.

Shiraiwa, M., Pfrang, C., Koop, T., and Pöschl, U.: Kinetic multi-layer model of gas-particle interactions in aerosols and clouds (KM-GAP): linking condensation, evaporation and chemical reactions of organics, oxidants and water, Atmospheric Chemistry and Physics, 12, 27772794, https://doi.org/10.5194/acp-12-2777-2012, https://www.atmos-chem-phys.net/12/2777/2012/, 2012.

Shiraiwa, M., Zuend, A., Bertram, A. K., and Seinfeld, J. H.: Gas-particle partitioning of atmospheric aerosols: interplay of physical state, non-ideal mixing and morphology, Phys. Chem. Chem. Phys., 15, 11 441-11453, https://doi.org/10.1039/C3CP51595H, http://dx.doi.org/ 10.1039/C3CP51595H, 2013.

Shiraiwa, M., Li, Y., Tsimpidi, A. P., Karydis, V. A., Berkemeier, T., Pandis, S. N., Lelieveld, J., Koop, T., and Pöschl, U.: Global distribution of particle phase state in atmospheric secondary organic aerosols, Nature communications, 8, 1-7, 2017.

Song, M., Marcolli, C., Krieger, U. K., Zuend, A., and Peter, T.: Liquid-liquid phase separation in aerosol particles: Dependence on O:C, organic functionalities, and compositional complexity, Geophysical Research Letters, 39, https://doi.org/10.1029/2012GL052807, https: //agupubs.onlinelibrary.wiley.com/doi/abs/10.1029/2012GL052807, 2012.

Song, M., Liu, P., Martin, S. T., and Bertram, A. K.: Liquid-liquid phase separation in particles containing secondary organic material free of inorganic salts, Atmospheric Chemistry and Physics, 17, 11 261-11 271, https://doi.org/10.5194/acp-17-11261-2017, https://www. atmos-chem-phys.net/17/11261/2017/, 2017.

Takahama, S. and Russell, L. M.: A molecular dynamics study of water mass accommodation on condensed phase water coated by fatty acid monolayers, Journal of Geophysical Research: Atmospheres, 116, https://doi.org/10.1029/2010JD014842, https://agupubs.onlinelibrary. wiley.com/doi/abs/10.1029/2010JD014842, 2011.

Takamuku, T., Tabata, M., Yamaguchi, A., Nishimoto, J., Kumamoto, M., Wakita, H., and Yamaguchi, T.: Liquid Structure of Acetonitrile-Water Mixtures by X-ray Diffraction and Infrared Spectroscopy, The Journal of Physical Chemistry B, 102, 8880-8888, https://doi.org/10.1021/jp9824297, https://doi.org/10.1021/jp9824297, 1998.

Taylor, R. S. and Garrett, B. C.: Accommodation of alcohols by the liquid/vapor interface of water: Molecular dynamics study, The Journal of Physical Chemistry B, 103, 844-851, 1999.

Torrie, G. and Valleau, J.: Nonphysical sampling distributions in Monte Carlo free-energy estimation: Umbrella sampling, Journal of Computational Physics, 23, 187-199, https://doi.org/https://doi.org/10.1016/0021-9991(77)90121-8, https://www.sciencedirect.com/science/ article/pii/0021999177901218, 1977. 
Tribello, G. A., Bonomi, M., Branduardi, D., Camilloni, C., and Bussi, G.: PLUMED 2: New Feathers for an Old Bird, Computer Physics

Truhlar, D. G. and Garrett, B. C.: Multidimensional Transition State Theory and the Validity of Grote- Hynes Theory, The Journal of Physical Chemistry B, 104, 1069-1072, 2000.

Tuckerman, M.: Statistical mechanics: theory and molecular simulation, Oxford university press, 2010.

Vargaftik, N., Volkov, B., and Voljak, L.: International tables of the surface tension of water, Journal of Physical and Chemical Reference Data, 12, 817-820, 1983.

Vieceli, J., Roeselová, M., Potter, N., Dang, L. X., Garrett, B. C., and Tobias, D. J.: Molecular Dynamics Simulations of Atmospheric Oxidants at the Air-Water Interface: Solvation and Accommodation of OH and O3, The Journal of Physical Chemistry B, 109, 15 87615 892, https://doi.org/10.1021/jp051361+, https://doi.org/10.1021/jp051361+, pMID: 16853017, 2005.

Voigtländer, J., Stratmann, F., Niedermeier, D., Wex, H., and Kiselev, A.: Mass accommodation coefficient of water: A combined computational fluid dynamics and experimental data analysis, Journal of Geophysical Research: Atmospheres, 112, https://doi.org/10.1029/2007JD008604, https://agupubs.onlinelibrary.wiley.com/doi/abs/10.1029/2007JD008604, 2007.

von Domaros, M., Lakey, P. S. J., Shiraiwa, M., and Tobias, D. J.: Multiscale Modeling of Human Skin Oil-Induced Indoor Air Chemistry: Combining Kinetic Models and Molecular Dynamics, The Journal of Physical Chemistry B, 124, 3836-3843, https://doi.org/10.1021/acs.jpcb.0c02818, https://doi.org/10.1021/acs.jpcb.0c02818, pMID: 32290653, 2020.

910 Ward, C.: Liquid-Vapour Phase Change Rates and Interfacial Entropy Production, Journal of Non-Equilibrium Thermodynamics, 27, 289303, https://doi.org/10.1515/JNETDY.2002.017, 2002.

Wendler, K., Thar, J., Zahn, S., and Kirchner, B.: Estimating the Hydrogen Bond Energy, The Journal of Physical Chemistry A, 114, 95299536, https://doi.org/10.1021/jp103470e, https://doi.org/10.1021/jp103470e, pMID: 20707378, 2010.

You, Y., Smith, M. L., Song, M., Martin, S. T., and Bertram, A. K.: Liquid-liquid phase separation in atmospherically relevant particles consisting of organic species and inorganic salts, International Reviews in Physical Chemistry, 33, 43-77, 2014.

Zientara, M., Jakubczyk, D., Kolwas, K., and Kolwas, M.: Temperature Dependence of the Evaporation Coefficient of Water in Air and Nitrogen under Atmospheric Pressure: Study in Water Droplets, The Journal of Physical Chemistry A, 112, 5152-5158, https://doi.org/10.1021/jp7114324, https://doi.org/10.1021/jp7114324, pMID: 18491849, 2008. 